\title{
CERTAIN BIPOLAR NEUTROSOPHIC COMPETITION GRAPHS
}

\author{
Muhammad Akram ${ }^{1}$ ANd Maryam NAsiR ${ }^{2}$ \\ ${ }^{1,2}$ Department of Mathematics, University of the Punjab, New Campus, \\ Lahore, Pakistan \\ makrammath@yahoo.com
}

\begin{abstract}
Bipolarity plays an important role in many research domains. A bipolar fuzzy model is a very important model in which positive information represents what is possible or preferred, while negative information represents what is forbidden or surely false. In this research paper, we first introduce the concept of $p$-competition bipolar neutrosophic graphs. We then define generalization of bipolar neutrosophic competition graphs called $m$-step bipolar neutrosophic competition graphs. Moreover, we present some related concepts of bipolar neutrosophic graphs. Finally, we describe an application of $m$-step bipolar neutrosophic competition graphs.

Key words and Phrases: $p$-competition bipolar neutrosophic graphs, $m$-step bipolar neutrosophic competition graphs, Algorithm.
\end{abstract}

\begin{abstract}
Abstrak. Bipolariti memainkan peran penting dalam berbagai macam topik penelitian. Sebuah model Fuzzy bipolar adalah sebuah model yang sangat penting dalam hal informasi postif menyatakan apa yang mungkin dipilih, sedangkan informasi negatif menyatakan apa yang dilarang atau pasti salah. Pada paper ini, pertama kali kami memperkenalkan konsep graf neotrosopik bipolar $p$-kompetisi. Kemudian kami mendefinisikan perumuman dari graf kompetisi neutrosopik bipolar yang disebut dengan graf kompetisi neutrosopik bipolar $m$-langkah. Lebih jauh, kami menyajikan beberapa konsep yang terkait dengan graf neutrosopik bipolar. Akhirnya, kami menggambarkan sebuah aplikasi dari graf kompetisi neutrosopik bipolar $m$-langkah.

Kata kunci: Graf neotrosopik bipolar p-kompetisi, graf kompetisi neutrosopik bipolar $m$-langkah, algoritma.
\end{abstract}

2000 Mathematics Subject Classification: 03E72, 68R10, 68R05. Received: 28-02-2017, revised: 11-04-2017, accepted: 09-05-2017. 


\section{INTRODUCTION}

The notion of competition graphs was introduced by Cohen [10] in 1968, depending upon a problem in ecology. The competition graphs have many utilizations in solving daily life problems, including channel assignment, modeling of complex economic, phytogenetic tree reconstruction, coding and energy systems.

Fuzzy set theory [26] and intuitionistic fuzzy set theory [6] are useful models for dealing with uncertainty and incomplete information. But they may not be sufficient in modeling of indeterminate and inconsistent information encountered in real world. In order to cope with this issue, neutrosophic set theory was proposed by Smarandache [18] as a generalization of fuzzy sets and intuitionistic fuzzy sets. However, since neutrosophic sets are identified by three functions called truthmembership $(t)$, indeterminacy-membership $(i)$ and falsity-membership $(f)$ whose values are real standard or non-standard subset of unit interval $] 0^{-}, 1^{+}[$. There are some difficulties in modeling of some problems in engineering and sciences. To overcome these difficulties, in 2010, concept of single-valued neutrosophic sets and its operations defined by Wang et al. [22] as a generalization of intuitionistic fuzzy sets. Ye $[24,25]$ has presented several novel applications of neutrosophic sets.

Deli et al. [11] extended the ideas of bipolar fuzzy sets [28] and neutrosophic sets to bipolar neutrosophic sets and studied its operations and applications in decision making problems. Smarandache [20] proposed notion of neutrosophic graph and they separated them to four main categories. Wu [23] discussed fuzzy digraphs. The concept of fuzzy $k$-competition graphs and $p$-competition fuzzy graphs was first introduced by Samanta and Pal in [15], it was further studied in [5, 13, 16, 17]. Cho et al. [9] proposed the generalization of a digraphs known as $m$-step competition graphs. Samanta et al. [16] introduced the generalization of fuzzy competition graphs, called $m$-step fuzzy competition graphs. On the other hand, the concepts of bipolar fuzzy competition graphs and intuitionistic fuzzy competition graphs are discussed in [17, 13]. Samanta et al. [16] also introduced the concepts of fuzzy $m$ step neighbouthood graphs. The notion of bipolar fuzzy graphs was first introduced by Akram [1] in 2011 as a generalization of fuzzy graphs. On the other hand, Akram and Shahzadi [4] first introduced the notion of neutrosophic soft graphs and gave its applications. Akram [2] introduced the notion of single-valued neutrosophic planar graphs. Akram and Sarwar have shown that there are some flaws in Broumi et al. [8] 's definition, which cannot be applied in network models. All the predator-prey relations cannot only be represented by bipolar neutrosophic competition graphs. For example, in a food web, species may have a chain consisting of same number of preys by which they can reach to their common preys. This idea motivates the necessity of $m$-step bipolar neutrosophic competition graphs. In this research paper, we first introduce the concept of $p$-competition bipolar neutrosophic graphs. We then define generalization of bipolar neutrosophic competition graphs called $m$-step bipolar neutrosophic competition graphs. Moreover, we present some related concepts of bipolar neutrosophic graphs. Finally, we describe an application of $m$-step bipolar neutrosophic competition graphs. 


\section{Certain Bipolar Neutrosophic Competition Graphs}

Definition 2.1. [26, 27]A fuzzy set $\mu$ in a universe $X$ is a mapping $\mu: X \rightarrow[0,1]$. A fuzzy relation on $X$ is a fuzzy set $\nu$ in $X \times X$.

Definition 2.2. [28]A bipolar fuzzy set on a non-empty set $X$ has the form

$$
A=\left\{\left(x, \mu_{A}^{P}(x), \mu_{A}^{N}(x)\right): x \in X\right\}
$$

where, $\mu_{A}^{P}: X \rightarrow[0,1]$ and $\mu_{A}^{N}: X \rightarrow[-1,0]$ are mappings. The positive membership value $\mu_{A}^{P}(x)$ represents the strength of truth or satisfaction of an element $x$ to a certain property corresponding to bipolar fuzzy set $A$ and $\mu_{A}^{N}(x)$ denotes the strength of satisfaction of an element $x$ to some counter property of bipolar fuzzy set $A$. If $\mu_{A}^{P}(x) \neq 0$ and $\mu_{A}^{N}(x)=0$ it is the situation when $x$ has only truth satisfaction degree for property $A$. If $\mu_{A}^{N}(x) \neq 0$ and $\mu_{A}^{P}(x)=0$, it is the case that $x$ is not satisfying the property of $A$ but satisfying the counter property to $A$. It is possible for $x$ that $\mu_{A}^{P}(x) \neq 0$ and $\mu_{A}^{N}(x) \neq 0$ when $x$ satisfies the property of $A$ as well as its counter property in some part of $X$.

Definition 2.3. [1]Let $X$ be a non-empty set. A mapping $B=\left(\mu_{B}^{P}, \mu_{B}^{N}\right): X \times$ $X \rightarrow[0,1] \times[-1,0]$ is a bipolar fuzzy relation on $X$ such that $\mu_{B}^{P}(x y) \in[0,1]$ and $\mu_{B}^{N}(x y) \in[-1,0]$ for $x, y \in X$.

Definition 2.4. [1] A bipolar fuzzy graph on $X$ is a pair $G=(A, B)$ where $A=$ $\left(\mu_{A}^{P}, \mu_{A}^{N}\right)$ is a bipolar fuzzy set on $X$ and $B=\left(\mu_{B}^{P}, \mu_{B}^{N}\right)$ is a bipolar fuzzy relation in $X$ such that

$$
\mu_{B}^{P}(x y) \leq \mu_{A}^{P}(x) \wedge \mu_{A}^{P}(y) \text { and } \mu_{B}^{N}(x y) \geq \mu_{A}^{N}(x) \vee \mu_{A}^{N}(y) \text { for all } x, y \in X .
$$

Definition 2.5. [21] A neutrosophic set $A$ on a non-empty set $X$ is characterized by a truth-membership fuction $t_{A}: X \rightarrow[0,1]$, indeterminacy-membership function $i_{A}: X \rightarrow[0,1]$ and a falsity-membership function $f_{A}: X \rightarrow[0,1]$. There is no restriction on the sum of $t_{A}(x), i_{A}(x)$ and $f_{A}(x)$ for all $x \in X$.

Definition 2.6. [11]A bipolar neutrosophic set $A$ on a non-empty set $X$ is an object of the form

$$
A=\left\{\left(x, t_{A}^{P}(x), i_{A}^{P}(x), f_{A}^{P}(x), t_{A}^{N}(x), i_{A}^{N}(x), f_{A}^{N}(x)\right): x \in X\right\},
$$

where $t_{A}^{P}, i_{A}^{P}, f_{A}^{P}: X \rightarrow[0,1]$ and $t_{A}^{N}, i_{A}^{N}, f_{A}^{N}: X \rightarrow[-1,0]$. The positive values $t_{A}^{P}(x), i_{A}^{P}(x), f_{A}^{P}(x)$ denote respectively the truth, indeterminacy and falsememberships degrees of an element $x \in X$, whereas, $t_{A}^{N}(x), i_{A}^{N}(x), f_{A}^{N}(x)$ denote the implicit counter property of the truth,indeterminacy and false-memberships degrees of the element $x \in X$ corresponding to the bipolar neutrosophic set $A$. 
Definition 2.7. The height of bipolar neutrosophic set $A=\left(t_{A}^{P}(x), i_{A}^{P}(x), f_{A}^{P}(x)\right.$, $\left.t_{A}^{N}(x), i_{A}^{N}(x), f_{A}^{N}(x)\right)$ in universe of discourse $X$ is defined as,

$$
\begin{aligned}
h(A) & =\left(h_{1}(A), h_{2}(A), h_{3}(A), h_{4}(A), h_{5}(A), h_{6}(A)\right) \\
& =\left(\sup _{x \in X} t_{A}^{P}(x), \sup _{x \in X} i_{A}^{P}(x), \inf _{x \in X} f_{A}^{P}(x), \sup _{x \in X} t_{A}^{P}(x), \sup _{x \in X} i_{A}^{P}(x), \inf _{x \in X} f_{A}^{P}(x)\right),
\end{aligned}
$$

for all $x \in X$.

Definition 2.8. Let $\vec{G}$ be a bipolar neutrosophic digraph then bipolar neutrosophic out-neighbourhoods of a vertex $x$ is a bipolar neutrosophic set

$$
\mathcal{N}^{+}(x)=\left(X_{x}^{+}, t_{x}^{(P)^{+}}, i_{x}^{(P)^{+}}, f_{x}^{(P)^{+}}, t_{x}^{(N)^{+}}, i_{x}^{(N)^{+}}, f_{x}^{(N)^{+}}\right),
$$

where,

$$
\begin{gathered}
X_{x}^{+}=\left\{y \mid B_{1}^{P} \overrightarrow{(x, y)}>0, B_{2}^{P} \overrightarrow{(x, y)}>0, B_{3}^{P} \overrightarrow{(x, y)}>0, B_{1}^{N} \overrightarrow{(x, y)}<0, B_{2}^{N} \overrightarrow{(x, y)}<0,\right. \\
\left.B_{3}^{N} \overrightarrow{(x, y)}<0\right\},
\end{gathered}
$$

such that $t_{x}^{(P)^{+}}: X_{x}^{+} \rightarrow[0,1]$, defined by $t_{x}^{(P)^{+}}(y)=B_{1}^{P} \overrightarrow{(x, y)}, i_{x}^{(P)^{+}}: X_{x}^{+} \rightarrow[0,1]$, defined by $i_{x}^{(P)^{+}}(y)=B_{2}^{P} \overrightarrow{(x, y)}, f_{x}^{(P)^{+}}: X_{x}^{+} \rightarrow[0,1]$, defined by $f_{x}^{(P)^{+}}(y)=$ $B_{3}^{P} \overrightarrow{(x, y)}, t_{x}^{(N)^{+}}: X_{x}^{+} \rightarrow[-1,0]$, defined by $t_{x}^{(N)^{+}}(y)=B_{1}^{N} \overrightarrow{(x, y)}, i_{x}^{(N)^{+}}: X_{x}^{+} \rightarrow$ $[-1,0]$, defined by $i_{x}^{(N)^{+}}(y)=B_{2}^{N} \overrightarrow{(x, y)}, f_{x}^{(N)^{+}}: X_{x}^{+} \rightarrow[-1,0]$, defined by $f_{x}^{(N)^{+}}(y)=$ $B_{3}^{N} \overrightarrow{(x, y)}$

Definition 2.9. Let $\vec{G}$ be a bipolar neutrosophic digraph then bipolar neutrosophic in-neighbourhoods of a vertex $x$ is a bipolar neutrosophic set

$$
\mathcal{N}^{-}(x)=\left(X_{x}^{-}, t_{x}^{(P)^{-}}, i_{x}^{(P)^{-}}, f_{x}^{(P)^{-}}, t_{x}^{(N)^{-}}, i_{x}^{(N)^{-}}, f_{x}^{(N)^{-}}\right),
$$

where,

$$
\begin{gathered}
X_{x}^{-}=\left\{y \mid B_{1}^{P} \overrightarrow{(y, x)}>0, B_{2}^{P} \overrightarrow{(y, x)}>0, B_{3}^{P} \overrightarrow{(y, x)}>0, B_{1}^{N} \overrightarrow{(y, x)}<0, B_{2}^{N} \overrightarrow{(y, x)}<0,\right. \\
\left.B_{3}^{N} \overrightarrow{(y, x)}<0\right\},
\end{gathered}
$$

such that $t_{x}^{(P)^{-}}: X_{x}^{-} \rightarrow[0,1]$, defined by $t_{x}^{(P)^{-}}(y)=B_{1}^{P} \overrightarrow{(y, x)}, i_{x}^{(P)^{-}}: X_{x}^{-} \rightarrow[0,1]$, defined by $i_{x}^{(P)^{-}}(y)=B_{2}^{P} \overrightarrow{(y, x)}, f_{x}^{(P)^{-}}: X_{x}^{-} \rightarrow[0,1]$, defined by $f_{x}^{(P)^{-}}(y)=$ $B_{3}^{P} \overrightarrow{(y, x)}, t_{x}^{(N)^{-}}: X_{x}^{-} \rightarrow[-1,0]$, defined by $t_{x}^{(N)^{-}}(y)=B_{1}^{N} \overrightarrow{(y, x)}, i_{x}^{(N)^{-}}: X_{x}^{-} \rightarrow$ $[-1,0]$, defined by $i_{x}^{(N)^{-}}(y)=B_{2}^{N} \overrightarrow{(y, x)}, f_{x}^{(N)^{-}}: X_{x}^{-} \rightarrow[-1,0]$, defined by $f_{x}^{(N)^{-}}(y)=$ $B_{3}^{N} \overrightarrow{(y, x)}$.

Definition 2.10. A bipolar neutrosophic competition graph of a bipolar neutrosophic graph $\vec{G}=(A, \vec{B})$ is an undirected bipolar neutrosophic graph $\overrightarrow{\mathcal{C}(G)}=$ $(A, R)$ which has the same vertex set as in $\vec{G}$ and there is an edge between two 
vertices $x$ and $y$ if and only if $\mathcal{N}^{+}(x) \cap \mathcal{N}^{+}(y)$ is non-empty. The positive truthmembership, indeterminacy-membership, falsity-membership and negative truthmembership, indeterminacy-membership, falsity-membership values of the edge $(x, y)$ are defined as,

(1) $t_{R}^{P}(x, y)=\left(t_{A}^{P}(x) \wedge t_{A}^{P}(y)\right) h_{1}\left(\mathcal{N}^{+}(x) \cap \mathcal{N}^{+}(y)\right)$,

(2) $i_{R}^{P}(x, y)=\left(i_{A}^{P}(x) \wedge i_{A}^{P}(y)\right) h_{2}\left(\mathcal{N}^{+}(x) \cap \mathcal{N}^{+}(y)\right)$,

(3) $f_{R}^{P}(x, y)=\left(f_{A}^{P}(x) \vee f_{A}^{P}(y)\right) h_{3}\left(\mathcal{N}^{+}(x) \cap \mathcal{N}^{+}(y)\right)$,

(4) $t_{R}^{N}(x, y)=\left(t_{A}^{N}(x) \vee t_{A}^{N}(y)\right) h_{4}\left(\mathcal{N}^{+}(x) \cap \mathcal{N}^{+}(y)\right)$,

(5) $i_{R}^{N}(x, y)=\left(i_{A}^{N}(x) \vee i_{A}^{N}(y)\right) h_{5}\left(\mathcal{N}^{+}(x) \cap \mathcal{N}^{+}(y)\right)$,

(6) $f_{R}^{N}(x, y)=\left(f_{A}^{N}(x) \wedge f_{A}^{N}(y)\right) h_{6}\left(\mathcal{N}^{+}(x) \cap \mathcal{N}^{+}(y)\right)$, for all $x, y \in X$.

Example 2.11. Consider $\vec{G}=(A, B)$ is a bipolar single-valued neutrosophic digraph, such that, $X=\{a, b, c, d\}, A=\{(a, 0.3,0.8,0.2,-0.5,-0.2,-0.1),(b$, $0.8,0.3,0.1,-0.5,-0.4,-0.2),(c, 0.4,0.5,0.6,-0.2,-0.3,-0.5),(d, 0.7,0.3,0.4$, $-0.2,-0.3,-0.5)\}$, and $B=\{(\overrightarrow{(a, b)}, 0.2,0.1,0.1,-0.4,-0.1,-0.2),(\overrightarrow{(a, c)}, 0.3$, $0.5,0.6,-0.2,-0.2,-0.1),(\overrightarrow{(b, d)}, 0.6,0.2,0.2,-0.1,-0.2,-0.3),(\overrightarrow{(d, c)}, 0.2,0.2$, $0.2,-0.2,-0.3,-0.5)\}$ as shown in Fig. 1 .

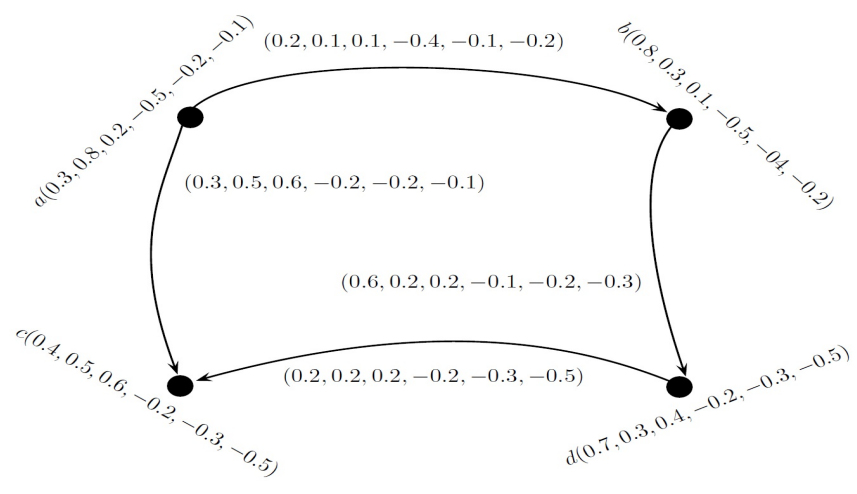

FigURE 1. Bipolar single-valued neutrosophic digraph

By direct calculations we have Table 1 representing bipolar single-valued neutrosophic out-neighbourhoods.

TABLE 1. Bipolar single-valued neutrosophic out-neighbourhoods

\begin{tabular}{|l|l|}
\hline$x$ & $\mathcal{N}^{+}(x)$ \\
\hline $\mathrm{a}$ & $\{(\mathrm{b}, 0.2,0.1,0.1,-0.4,-0.1,-0.2),(\mathrm{c}, 0.3,0.5,0.6,-0.2,-0.2,-0.1)\}$ \\
$\mathrm{b}$ & $\{(\mathrm{d}, 0.6,0.2,0.2,-0.1,-0.2,-0.3)\}$ \\
$\mathrm{c}$ & $\emptyset$ \\
$\mathrm{d}$ & $\{(\mathrm{c}, 0.2,0.2,0.2,-0.2,-0.3,-0.5)\}$ \\
\hline
\end{tabular}


Then bipolar single-valued neutrosophic competition graph of Fig. 1 is shown in Fig. 2.

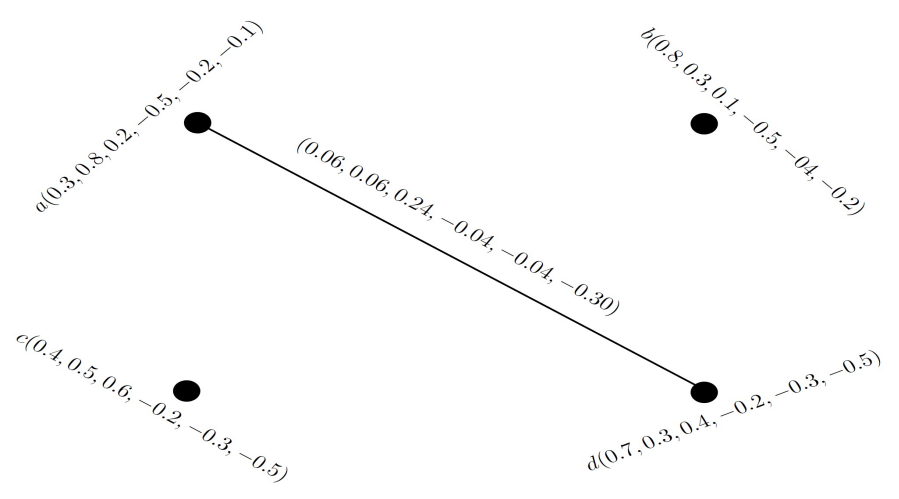

FiguRE 2. Bipolar single-valued neutrosophic competition graph

Definition 2.12. The support of a bipolar neutrosophic set $A=\left(x, t_{A}^{P}, i_{A}^{P}, f_{A}^{P}\right.$, $\left.t_{A}^{N}, i_{A}^{N}, f_{A}^{N}\right)$ in $X$ is the subset of $X$ defined by

$$
\begin{gathered}
\operatorname{supp}(A)=\left\{x \in X: t_{A}^{P}(x) \neq 0, i_{A}^{P}(x) \neq 0, f_{A}^{P}(x) \neq 1, t_{A}^{N}(x) \neq-1, i_{A}^{N}(x) \neq-1,\right. \\
\left.f_{A}^{N}(x) \neq 0\right\}
\end{gathered}
$$

and $|\operatorname{supp}(A)|$ is the number of elements in the set.

Example 2.13. The support of a bipolar neutrosophic set $A=\{(a, 0.5,0.7,0.2$, $-0.8,-0.9,-0.3),(b, 0.1,0.2,1,-0.5,-0.7,-0.6),(c, 0.3,0.5,0.3,-0.8,-0.6$, $-0.4),(d, 0,0,1,-1,-1,0)\}$ in $X=\{a, b, c, d\}$ is $\operatorname{supp}(A)=\{a, b, c\}$ and $|\operatorname{supp}(A)|=3$.

We now discuss $p$-competition bipolar neutrosophic graphs.

Definition 2.14. Let $p$ be a positive integer. Then $p$-competition bipolar neutrosophic graph $\mathcal{C}^{p}(\vec{G})$ of the bipolar neutrosophic digraph $\vec{G}=(A, \vec{B})$ is an undirected bipolar neutrosophic graph $G=(A, B)$ which has same bipolar neutrosophic set of vertices as in $\vec{G}$ and has a bipolar neutrosophic edge between two vertices $x, y \in X$ in $\mathcal{C}^{p}(\vec{G})$ if and only if $\left|\operatorname{supp}\left(\mathcal{N}^{+}(x) \cap \mathcal{N}^{+}(y)\right)\right| \geq p$. The positive truth-membership value of edge $(x, y)$ in $\mathcal{C}^{p}(\vec{G})$ is $t_{B}^{P}(x, y)=\frac{(i-p)+1}{i}\left[t_{A}^{P}(x) \wedge\right.$ $\left.t_{A}^{P}(y)\right] h_{1}\left(\mathcal{N}^{+}(x) \cap \mathcal{N}^{+}(y)\right)$, the positive indeterminacy-membership value of edge $(x, y)$ in $\mathcal{C}^{p}(\vec{G})$ is $i_{B}^{P}(x, y)=\frac{(i-p)+1}{i}\left[i_{A}^{P}(x) \wedge i_{A}^{P}(y)\right] h_{2}\left(\mathcal{N}^{+}(x) \cap \mathcal{N}^{+}(y)\right)$, positive falsity-membership value of edge $(x, y)$ in $\mathcal{C}^{p}(\vec{G})$ is $f_{B}^{P}(x, y)=\frac{(i-p)+1}{i}\left[f_{A}^{P}(x) \vee\right.$ $\left.f_{A}^{P}(y)\right] h_{3}\left(\mathcal{N}^{+}(x) \cap \mathcal{N}^{+}(y)\right)$, the negative truth-membership value of edge $(x, y)$ 
in $\mathcal{C}^{p}(\vec{G})$ is $t_{B}^{N}(x, y)=\frac{(i-p)+1}{i}\left[t_{A}^{N}(x) \vee t_{A}^{N}(y)\right] h_{4}\left(\mathcal{N}^{+}(x) \cap \mathcal{N}^{+}(y)\right)$, the negative indeterminacy-membership value of edge $(x, y)$ in $\mathcal{C}^{p}(\vec{G})$ is $i_{B}^{N}(x, y)=\frac{(i-p)+1}{i}\left[i_{A}^{N}(x) \vee\right.$ $\left.i_{A}^{N}(y)\right] h_{5}\left(\mathcal{N}^{+}(x) \cap \mathcal{N}^{+}(y)\right)$, negative falsity-membership value of edge $(x, y)$ in $\mathcal{C}^{p}(\vec{G})$ is $f_{B}^{N}(x, y)=\frac{(i-p)+1}{i}\left[f_{A}^{N}(x) \wedge f_{A}^{N}(y)\right] h_{6}\left(\mathcal{N}^{+}(x) \cap \mathcal{N}^{+}(y)\right)$, where $i=$ $\left|\operatorname{supp}\left(\mathcal{N}^{+}(x) \cap \mathcal{N}^{+}(y)\right)\right|$.

The 3-competition bipolar neutrosophic graph is illustrated by the following example.

Example 2.15. Consider $\vec{G}=(A, \vec{B})$ is a bipolar neutrosophic digraph, such that, $X=\{x, y, z, a, b, c\}, A=\{(x, 0.7,0.8,0.5,-0.5,-0.6,-0.7),(y, 0.6$, $0.7,0.5,-0.3,-0.2,-0.7),(z, 0.6,0.7,0.3,-0.2,-0.3,-0.4),(a, 0.5,0.6,0.7$, $-0.5,-0.6,-0.8),(b, 0.5,0.6,0.7,-0.9,-0.8,-0.7),(c, 0.5,0.6,0.3,-0.1,-0.2$, $-0.4)\}$, and $B=\{(\overrightarrow{(x, a)}, 0.3,0.4,0.6,-0.4,-0.5,-0.7),(\overrightarrow{(x, b)}, 0.4,0.5,0.4,-0.4$, $-0.5,-0.5),(\overrightarrow{(x, c)}, 0.4,0.5,0.4,-0.1,-0.1,-0.6),(\overrightarrow{(y, a)}, 0.4,0.5,0.6,-0.2,-0.2$, $-0.6),(\overrightarrow{(y, b)}, 0.4,0.4,0.6,-0.2,-0.2,-0.6),(\overrightarrow{(y, c)}, 0.4,0.5,0.4,-0.1,-0.2,-0.3)$, $(\overrightarrow{(z, b)}, 0.4,0.5,0.3,-0.1,-0.2,-0.6),(\overrightarrow{(z, c)}, 0.4,0.5,0.2,-0.1,-0.2,-0.3)\}$, as shown in Fig. 3. Then $\mathcal{N}^{+}(x)=\{(a, 0.3,0.4,0.6,-0.4,-0.5,-0.7),(b, 0.4,0.5$, $0.4,-0.4,-0.5,-0.5),(c, 0.4,0.5,0.4,-0.1,-0.1,-0.6)\}, \mathcal{N}^{+}(y)=\{(a, 0.4,0.5$, $0.6,-0.2,-0.2,-0.6),(b, 0.4,0.4,0.6,-0.2,-0.2,-0.6),(c, 0.4,0.5,0.4,-0.1$, $-0.2,-0.3)\}, \mathcal{N}^{+}(z)=\{(b, 0.4,0.5,0.3,-0.1,-0.2,-0.6),(c, 0.4,0.5,0.2,-0.1$, $-0.2,-0.3)\}$. So, $\mathcal{N}^{+}(x) \cap \mathcal{N}^{+}(y)=\{(a, 0.3,0.4,0.6,-0.2,-0.2,-0.7),(b, 0.4$, $0.4,0.6,-0.2,-0.2,-0.6),(c, 0.4,0.5,0.4,-0.1,-0.1,-0.6)\}$.

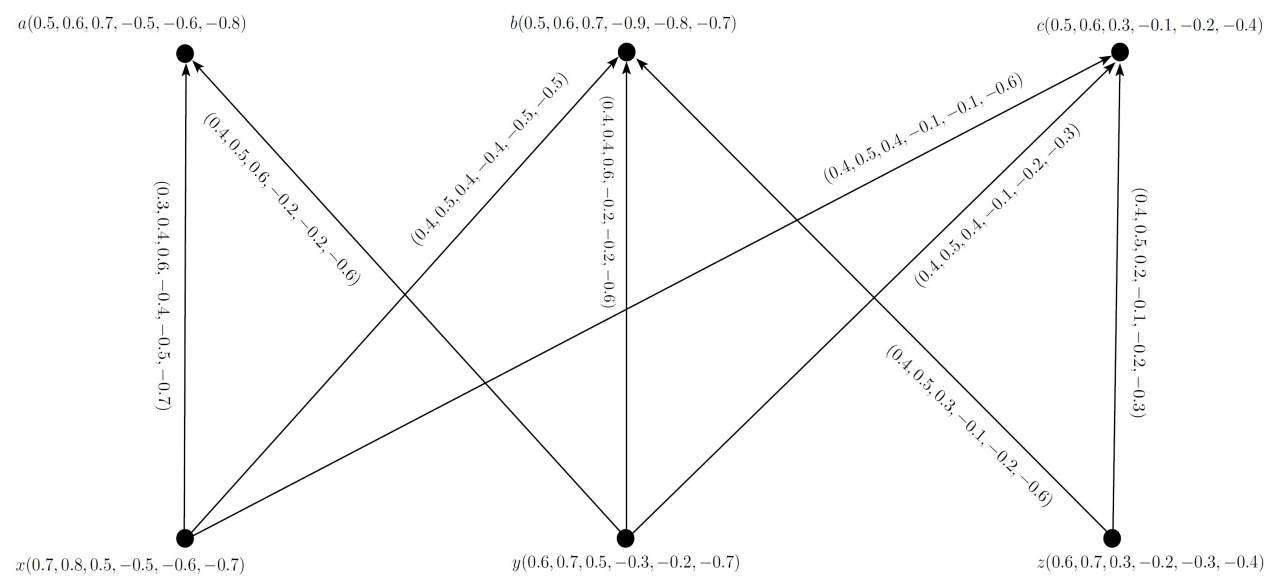

Figure 3. Bipolar neutrosophic digraph 
Now $i=\left|\operatorname{supp}\left(\mathcal{N}^{+}(x) \cap \mathcal{N}^{+}(y)\right)\right|=3$. For $p=3, t_{B}^{P}(x, y)=0.08, i_{B}^{P}(x$, $y)=0.1166, f_{B}^{P}(x, y)=0.066, t_{B}^{N}(x, y)=-0.04, i_{B}^{N}(x, y)=-0.033$, and $f_{B}^{N}(x$, $y)=-0.0933$. As shown in Fig. 4 .

$a(0.5,0.6,0.7,-0.5,-0.6,-0.8)$

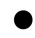

$b(0.5,0.6,0.7,-0.9,-0.8,-0.7)$

$(0.5,0.6,0.7,-1$ $c(0.5,0.6,0.3,-0.1,-0.2,-0.4)$

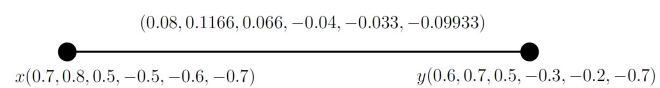

$(0.6,0.7,0.3,-0.2,-0.3$

FIGURE 4. 3-competition bipolar neutrosophic graph

Theorem 2.16. Let $\vec{G}=(A, \vec{B})$ be a bipolar neutrosophic digraph. If $h_{1}\left(\mathcal{N}^{+}(x) \cap \mathcal{N}^{+}(y)\right)=1, \quad h_{2}\left(\mathcal{N}^{+}(x) \cap \mathcal{N}^{+}(y)\right)=1, \quad h_{3}\left(\mathcal{N}^{+}(x) \cap \mathcal{N}^{+}(y)\right)=0$, $h_{4}\left(\mathcal{N}^{+}(x) \cap \mathcal{N}^{+}(y)\right)=1, \quad h_{5}\left(\mathcal{N}^{+}(x) \cap \mathcal{N}^{+}(y)\right)=1, \quad h_{6}\left(\mathcal{N}^{+}(x) \cap \mathcal{N}^{+}(y)\right)=0$,

in $\mathcal{C}^{\left[\frac{i}{2}\right]}(\vec{G})$, then the edge $(x, y)$ is strong, where $i=\left|\operatorname{supp}\left(\mathcal{N}^{+}(x) \cap \mathcal{N}^{+}(y)\right)\right|$. (Note that for any real number $x,[x]=$ greatest integer not exceeding $x$.)

PRoOF. Suppose $\vec{G}=(A, \vec{B})$ is a bipolar neutrosophic digraph. Let the corresponding $\left[\frac{i}{2}\right]$-bipolar neutrosophic competition graph be $\mathcal{C}^{\left[\frac{i}{2}\right]}(\vec{G})$, where $i=$ $\left|\operatorname{supp}\left(\mathcal{N}^{+}(x) \cap \mathcal{N}^{+}(y)\right)\right|$. Also, assume that

$h_{1}\left(\mathcal{N}^{+}(x) \cap \mathcal{N}^{+}(y)\right)=1, \quad h_{2}\left(\mathcal{N}^{+}(x) \cap \mathcal{N}^{+}(y)\right)=1, \quad h_{3}\left(\mathcal{N}^{+}(x) \cap \mathcal{N}^{+}(y)\right)=0$,

$h_{4}\left(\mathcal{N}^{+}(x) \cap \mathcal{N}^{+}(y)\right)=1, \quad h_{5}\left(\mathcal{N}^{+}(x) \cap \mathcal{N}^{+}(y)\right)=1, \quad h_{6}\left(\mathcal{N}^{+}(x) \cap \mathcal{N}^{+}(y)\right)=0$,

for all $x, y \in X$. Now,

$$
\begin{gathered}
t_{B}^{P}(x, y)=\frac{\left(i-\left[\frac{i}{2}\right]\right)+1}{i}\left[t_{A}^{P}(x) \wedge t_{A}^{P}(y)\right] \times 1, t_{B}^{N}(x, y)=\frac{\left(i-\left[\frac{i}{2}\right]\right)+1}{i}\left[t_{A}^{N}(x) \vee t_{A}^{N}(y)\right] \times 1, \\
i_{B}^{P}(x, y)=\frac{\left(i-\left[\frac{i}{2}\right]\right)+1}{i}\left[i_{A}^{P}(x) \wedge i_{A}^{P}(y)\right] \times 1, i_{B}^{N}(x, y)=\frac{\left(i-\left[\frac{i}{2}\right]\right)+1}{i}\left[i_{A}^{N}(x) \vee i_{A}^{N}(y)\right] \times 1, \\
f_{B}^{P}(x, y)=\frac{\left(i-\left[\frac{i}{2}\right]\right)+1}{i}\left[f_{A}^{P}(x) \vee f_{A}^{P}(y)\right] \times 0, f_{B}^{N}(x, y)=\frac{\left(i-\left[\frac{i}{2}\right]\right)+1}{i}\left[f_{A}^{N}(x) \wedge f_{A}^{N}(y)\right] \times 0 .
\end{gathered}
$$


This gives the result,

$$
\begin{array}{cl}
\frac{t_{B}^{P}(x, y)}{\left[t_{A}^{P}(x) \wedge t_{A}^{P}(y)\right]}=\frac{\left(i-\left[\frac{i}{2}\right]\right)+1}{i}>0.5, & \frac{t_{B}^{N}(x, y)}{\left[t_{A}^{N}(x) \vee t_{A}^{N}(y)\right]}=\frac{\left(i-\left[\frac{i}{2}\right]\right)+1}{i}<0.5, \\
\frac{i_{B}^{P}(x, y)}{\left[i_{A}^{P}(x) \wedge i_{A}^{P}(y)\right]}=\frac{\left(i-\left[\frac{i}{2}\right]\right)+1}{i}>0.5, & \frac{i_{B}^{N}(x, y)}{\left[i_{A}^{N}(x) \vee i_{A}^{N}(y)\right]}=\frac{\left(i-\left[\frac{i}{2}\right]\right)+1}{i}<0.5, \\
\frac{f_{B}^{P}(x, y)}{\left[f_{A}^{P}(x) \vee f_{A}^{P}(y)\right]}=\frac{\left(i-\left[\frac{i}{2}\right]\right)+1}{i}<0.5, & \frac{f_{B}^{N}(x, y)}{\left[f_{A}^{N}(x) \wedge f_{A}^{N}(y)\right]}=\frac{\left(i-\left[\frac{i}{2}\right]\right)+1}{i}<0.5 .
\end{array}
$$

Hence, the edge $(x, y)$ is strong. This proves the result.

We now define another extension of bipolar neutrosophic competition graph known as $m$-step bipolar neutrosophic competition graph.

In this paper, we will use the following notations:

$\mathcal{P}_{x, y}^{m}$ : A bipolar neutrosophic path of length $m$ from $x$ to $y$.

$\overrightarrow{\mathcal{P}}_{x, y}^{m}$ : A directed bipolar neutrosophic path of length $m$ from $x$ to $y$.

$\mathcal{N}_{m}^{+}(x): m$-step bipolar neutrosophic out-neighbourhood of vertex $x$.

$\mathcal{N}_{m}^{-}(x): m$-step bipolar neutrosophic in-neighbourhood of vertex $x$.

$\mathcal{N}_{m}(x): m$-step bipolar neutrosophic neighbourhood of vertex $x$.

$\mathcal{N}_{m}(G): m$-step bipolar neutrosophic neighbourhood graph of the bipolar neutrosophic graph $G$.

$\mathcal{C}_{m} \overrightarrow{(G)}: m$-step bipolar neutrosophic competition graph of the bipolar neutrosophic digraph $\vec{G}$.

Definition 2.17. Suppose $\vec{G}=(A, \vec{B})$ is a bipolar neutrosophic digraph. The $m$ step bipolar neutrosophic digraph of $\vec{G}$ is denoted by $\vec{G}_{m}=(A, B)$, where bipolar neutrosophic set of vertices of $\vec{G}$ is same with bipolar neutrosophic set of vertices of $\vec{G}_{m}$ and has an edge between $x$ and $y$ in $\vec{G}_{m}$ if and only if there exists a bipolar neutrosophic directed path $\overrightarrow{\mathcal{P}}_{x, y}^{m}$ in $\vec{G}$.

Definition 2.18. The bipolar neutrosophic $m$-step out-neighbourhood of vertex $x$ of a bipolar neutrosophic digraph $\vec{G}=(A, \vec{B})$ is bipolar neutrosophic set

$$
\mathcal{N}_{m}^{+}(x)=\left(X_{x}^{+}, t_{x}^{(P)^{+}}, i_{x}^{(P)^{+}}, f_{x}^{(P)^{+}}, t_{x}^{(N)^{+}}, i_{x}^{(N)^{+}}, f_{x}^{(N)^{+}}\right), \quad \text { where }
$$

$X_{x}^{+}=\{y \mid$ there exists a directed bipolar neutrosophic path of length $m$ from $x$ to $\left.y, \vec{P}_{x, y}^{m}\right\}, t_{x}^{(P)^{+}}: X_{x}^{+} \rightarrow[0,1], i_{x}^{(P)^{+}}: X_{z}^{+} \rightarrow[0,1], f_{x}^{(P)^{+}}: X_{z}^{+} \rightarrow[0,1]$ $t_{x}^{(N)^{+}}: X_{x}^{+} \rightarrow[-1,0], i_{x}^{(N)^{+}}: X_{z}^{+} \rightarrow[-1,0], f_{x}^{(N)^{+}}: X_{z}^{+} \rightarrow[-1,0]$ are defined by $t_{x}^{(P)^{+}}=\min \left\{t^{P} \overrightarrow{\left(x_{1}, x_{2}\right)},\left(x_{1}, x_{2}\right)\right.$ is an edge of $\left.\overrightarrow{\mathcal{P}}_{x, y}^{m}\right\}, i_{x}^{(P)^{+}}=\min \left\{i^{P} \overrightarrow{\left(x_{1}, x_{2}\right)},\left(x_{1}\right.\right.$, $\left.x_{2}\right)$ is an edge of $\left.\overrightarrow{\mathcal{P}}_{x, y}^{m}\right\}, f_{x}^{(P)^{+}}=\max \left\{f^{P} \overline{\left(x_{1}, x_{2}\right)},\left(x_{1}, x_{2}\right)\right.$ is an edge of $\left.\overrightarrow{\mathcal{P}}_{x, y}^{m}\right\}$, $t_{x}^{(N)^{+}}=\max \left\{t^{P} \overrightarrow{\left(x_{1}, x_{2}\right)},\left(x_{1}, x_{2}\right)\right.$ is an edge of $\left.\overrightarrow{\mathcal{P}}_{x, y}^{m}\right\}, i_{x}^{(N)^{+}}=\max \left\{i^{N} \overrightarrow{\left(x_{1}, x_{2}\right)}\right.$, 
$\left(x_{1}, x_{2}\right)$ is an edge of $\left.\overrightarrow{\mathcal{P}}_{x, y}^{m}\right\}, f_{x}^{(N)^{+}}=\min \left\{f^{N} \overrightarrow{\left(x_{1}, x_{2}\right)},\left(x_{1}, x_{2}\right)\right.$ is an edge of $\left.\overrightarrow{\mathcal{P}}_{x, y}^{m}\right\}$, respectively.

Example 2.19. Consider $\vec{G}=(A, \vec{B})$ is a bipolar neutrosophic digraph, such that $X=\{x, y, a, b, c, d\}$, as shown in Fig. 5. Then, 2-step out-neighbourhood of vertices $x$, and $y$ is calculated as, $\mathcal{N}_{2}^{+}(x)=\{(b, 0.2,0.2,0.5,-0.2,-0.3,-0.3),(d, 0.2$, $0.2,0.5,-0.2,-0.3,-0.3)\}, \mathcal{N}_{2}^{+}(y)=\{(b, 0.1,0.3,0.2,-0.2,-0.3,-0.6),(d, 0.3,0.5$, $0.6,-0.2,-0.3,-0.5)\}$.

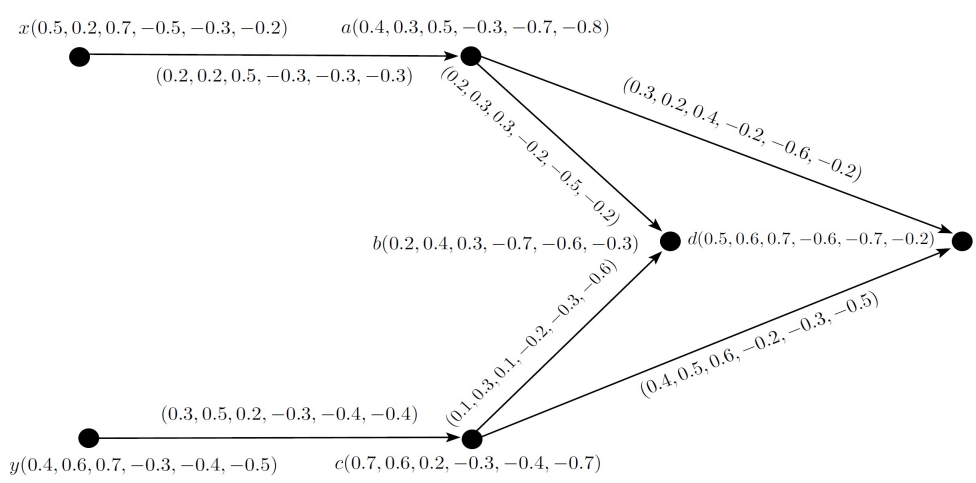

FiguRE 5. Bipolar neutrosophic digraph

Definition 2.20. The bipolar neutrosophic m-step in-neighbourhood of vertex $x$ of a bipolar neutrosophic digraph $\vec{G}=(A, \vec{B})$ is bipolar neutrosophic set

$$
\mathcal{N}_{m}^{-}(x)=\left(X_{x}^{-}, t_{x}^{(P)^{-}}, i_{x}^{(P)^{-}}, f_{x}^{(P)^{-}}, t_{x}^{(N)^{-}}, i_{x}^{(N)^{-}}, f_{x}^{(N)^{-}}\right) \text {, where }
$$

$X_{x}^{-}=\{y \mid$ there exists a directed bipolar neutrosophic path of length $m$ from $y$ to $\left.x, \vec{P}_{y, x}^{m}\right\}, t_{x}^{(P)^{-}}: X_{x}^{-} \rightarrow[0,1], i_{x}^{(P)^{-}}: X_{z}^{-} \rightarrow[0,1], f_{x}^{(P)^{-}}: X_{z}^{-} \rightarrow[0,1]$ $t_{x}^{(N)^{-}}: X_{x}^{-} \rightarrow[-1,0], i_{x}^{(N)^{-}}: X_{z}^{-} \rightarrow[-1,0], f_{x}^{(N)^{-}}: X_{z}^{-} \rightarrow[-1,0]$ are defined by $t_{x}^{(P)^{-}}=\min \left\{t^{P} \overrightarrow{\left(x_{1}, x_{2}\right)},\left(x_{1}, x_{2}\right)\right.$ is an edge of $\left.\overrightarrow{\mathcal{P}}_{y, x}^{m}\right\}, i_{x}^{(P)^{-}}=\min \left\{i^{P} \overrightarrow{\left(x_{1}, x_{2}\right)},\left(x_{1}\right.\right.$, $\left.x_{2}\right)$ is an edge of $\left.\overrightarrow{\mathcal{P}}_{y, x}^{m}\right\}, f_{x}^{(P)^{-}}=\max \left\{f^{P} \overline{\left(x_{1}, x_{2}\right.}\right),\left(x_{1}, x_{2}\right)$ is an edge of $\left.\overrightarrow{\mathcal{P}}_{y, x}^{m}\right\}$, $t_{x}^{(N)^{-}}=\max \left\{t^{N} \overrightarrow{\left(x_{1}, x_{2}\right)},\left(x_{1}, x_{2}\right)\right.$ is an edge of $\left.\overrightarrow{\mathcal{P}}_{y_{x}}^{m}\right\}, i_{x}^{(N)^{-}}=\max \left\{i^{N} \overrightarrow{\left(x_{1}, x_{2}\right)}\right.$, $\left(x_{1}, x_{2}\right)$ is an edge of $\left.\overrightarrow{\mathcal{P}}_{y, x}^{m}\right\}, f_{x}^{(N)^{-}}=\min \left\{f^{N} \overrightarrow{\left(x_{1}, x_{2}\right)},\left(x_{1}, x_{2}\right)\right.$ is an edge of $\left.\overrightarrow{\mathcal{P}}_{y, x}^{m}\right\}$, respectively.

Example 2.21. Consider $\vec{G}=(A, \vec{B})$ is a bipolar neutrosophic digraph, such that, $X=\{a, b, c, d, e, f\}$, as shown in Fig. 6. Then, 2-step in-neighbourhood of vertices $a$, and $b$ is calculated as, $\mathcal{N}_{2}^{-}(a)=\{(f, 0.1,0.1,0.5,-0.1,-0.2,-0.6),(e$, 
$0.3,0.1,0.7,-0.1,-0.2,-0.4)\}, \mathcal{N}_{2}^{-}(b)=\{(f, 0.1,0.3,0.6,-0.3,-0.4,-0.7),(e$, $0.4,0.3,0.6,-0.3,-0.4,-0.5)\}$.

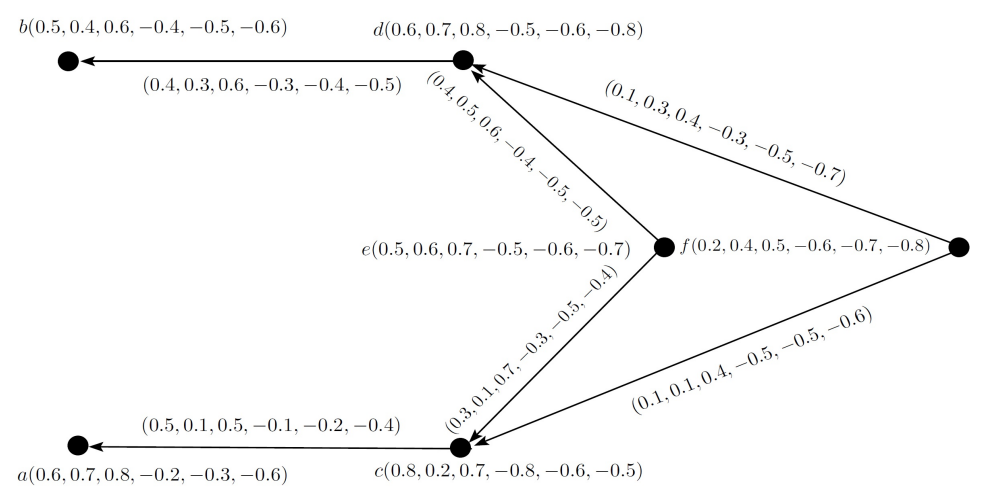

FIGURE 6. Bipolar neutrosophic digraph

Definition 2.22. Suppose $\vec{G}=(A, \vec{B})$ is a bipolar neutrosophic digraph. The $m$-step bipolar neutrosophic competition graph of bipolar neutrosophic digraph $\vec{G}$ is denoted by $\mathcal{C}_{m}(\vec{G})=(A, B)$ which has same bipolar neutrosophic set of vertices as in $\vec{G}$ and has an edge between two vertices $x, y \in X$ in $\mathcal{C}_{m}(\vec{G})$ if and only if $\left(\mathcal{N}_{m}^{+}(x) \cap \mathcal{N}_{m}^{+}(y)\right)$ is a non-empty bipolar neutrosophic set in $\vec{G}$. The positive truth-membership value of edge $(x, y)$ in $\mathcal{C}_{m}(\vec{G})$ is $t_{B}^{P}(x, y)=\left[t_{A}^{P}(x) \wedge\right.$ $\left.t_{A}^{P}(z)\right] h_{1}\left(\mathcal{N}_{m}^{+}(x) \cap \mathcal{N}_{m}^{+}(y)\right)$, the positive indeterminacy-membership value of edge $(x$, $y)$ in $\mathcal{C}_{m}(\vec{G})$ is $i_{B}^{P}(x, y)=\left[i_{A}^{P}(x) \wedge i_{A}^{P}(y)\right] h_{2}\left(\mathcal{N}_{m}^{+}(x) \cap \mathcal{N}_{m}^{+}(y)\right)$, the positive falsitymembership value of edge $(x, y)$ in $\mathcal{C}_{m}(\vec{G})$ is $f_{B}^{P}(x, y)=\left[f_{A}^{P}(x) \vee f_{A}^{P}(y)\right] h_{3}\left(\mathcal{N}_{m}^{+}(x) \cap\right.$ $\left.\mathcal{N}_{m}^{+}(y)\right)$, the negative truth-membership value of edge $(x, y)$ in $\mathcal{C}_{m}(\vec{G})$ is $t_{B}^{N}(x$, $y)=\left[t_{A}^{N}(x) \vee t_{A}^{N}(z)\right] h_{4}\left(\mathcal{N}_{m}^{+}(x) \cap \mathcal{N}_{m}^{+}(y)\right)$, the negative indeterminacy-membership value of edge $(x, y)$ in $\mathcal{C}_{m}(\vec{G})$ is $i_{B}^{N}(x, y)=\left[i_{A}^{N}(x) \vee i_{A}^{N}(y)\right] h_{5}\left(\mathcal{N}_{m}^{+}(x) \cap \mathcal{N}_{m}^{+}(y)\right)$, the negative falsity-membership value of edge $(x, y)$ in $\mathcal{C}_{m}(\vec{G})$ is $f_{B}^{N}(x, y)=$ $\left[f_{A}^{N}(x) \wedge f_{A}^{N}(y)\right] h_{6}\left(\mathcal{N}_{m}^{+}(x) \cap \mathcal{N}_{m}^{+}(y)\right)$.

The 2-step bipolar neutrosophic competition graph is illustrated by the following example.

Example 2.23. Consider $\vec{G}=(A, \vec{B})$ is a bipolar neutrosophic digraph, such that $X=\{x, y, a, b, c, d\}$, as shown in Fig. 7. Then, $\mathcal{N}_{2}^{+}(x)=\{(b, 0.2,0.2,0.5$, $-0.2,-0.3,-0.3),(d, 0.2,0.2,0.5,-0.2,-0.3,-0.3)\}, \mathcal{N}_{2}^{+}(y)=\{(b, 0.1,0.3,0.2$, $-0.2,-0.3,-0.6),(d, 0.3,0.5,0.6,-0.2,-0.3,-0.5)\}$, there non-empty intersection 


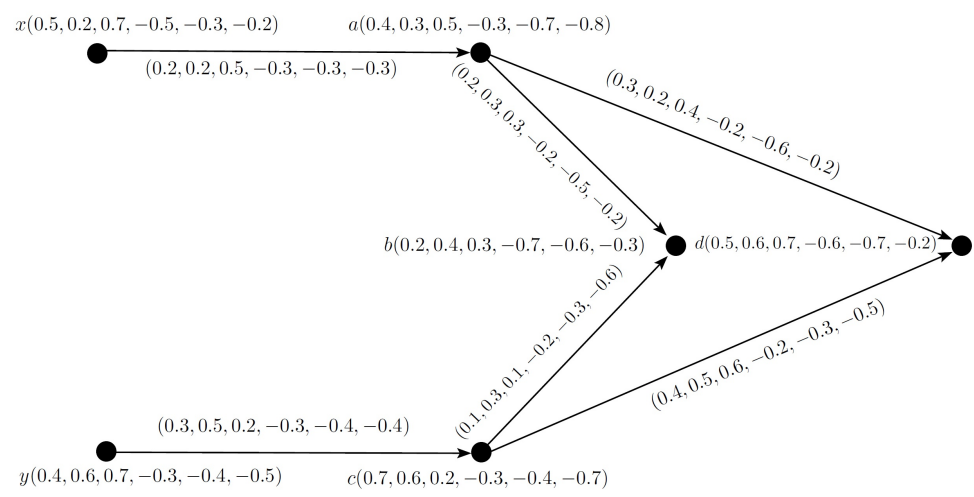

FIGURE 7. Bipolar neutrosophic digraph

is calculated as $\mathcal{N}_{2}^{+}(x) \cap \mathcal{N}_{2}^{+}(y)=\{(b, 0.1,0.2,0.5,-0.2,-0.3,-0.6),(d, 0.2,0.2$, $0.6,-0.2,-0.3,-0.5)\}$.

Thus, $t_{B}^{P}(x, y)=0.08, i_{B}^{P}(x, y)=0.04, f_{B}^{P}(x, y)=0.35, t_{B}^{N}(x, y)=-0.06$, $i_{B}^{N}(x, y)=-0.06$, and $f_{B}^{N}(x, y)=-0.25$. Then its corresponding 2-step bipolar neutrosophic competition graph is shown in Fig. 8.

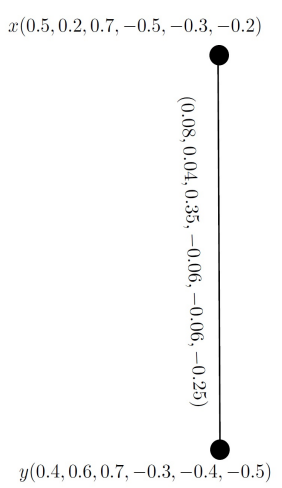

$a(0.4,0.3,0.5,-0.3,-0.7,-0.8)$

$b(0.2,0.4,0.3,-0.7,-0.6,-0.3)$

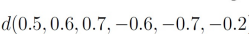

FIGURE 8. 2-step bipolar neutrosophic competition graph

If a predator $x$ attacks one prey $y$, then the linkage is shown by an edge $\overrightarrow{(x, y)}$ in a bipolar neutrosophic digraph. But, if predator needs help of many other mediators $x_{1}, x_{2}, \ldots x_{m-1}$, then linkage among them is shown by bipolar neutrosophic directed path $\overrightarrow{\mathcal{P}}_{x, y}^{m}$ in a bipolar neutrosophic digraph. So, $m$-step prey in a bipolar neutrosophic digraph is represented by a vertex which is the $m$-step out-neighbourhood of some vertices. Now, the strength of a bipolar neutrosophic competition graphs is defined below. 
Definition 2.24. Let $\vec{G}=(A, \vec{B})$ be a bipolar neutrosophic digraph. Let $w$ be a common vertex of $m$-step out-neighbourhoods of vertices $x_{1}, x_{2}, \ldots, x_{l}$. Also, let $\overrightarrow{B_{1}^{P}}\left(u_{1}, v_{1}\right), \overrightarrow{B_{1}^{P}}\left(u_{2}, v_{2}\right), \ldots, \overrightarrow{B_{1}^{P}}\left(u_{l}, v_{l}\right)$ be the minimum positive truthmembership values, $\overrightarrow{B_{2}^{P}}\left(u_{1}, v_{1}\right), \overrightarrow{B_{2}^{P}}\left(u_{2}, v_{2}\right), \ldots, \overrightarrow{B_{2}^{P}}\left(u_{l}, v_{l}\right)$ be the minimum positive indeterminacy-membership values, $\overrightarrow{B_{3}^{P}}\left(u_{1}, v_{1}\right), \overrightarrow{B_{3}^{P}}\left(u_{2}, v_{2}\right) \ldots, \overrightarrow{B_{3}^{P}}\left(u_{l}, v_{l}\right)$ be the maximum positive false-membership values, $\overrightarrow{B_{1}^{N}}\left(u_{1}, v_{1}\right), \overrightarrow{B_{1}^{N}}\left(u_{2}, v_{2}\right), \ldots, \overrightarrow{B_{1}^{N}}\left(u_{l}, v_{l}\right)$ be the maximum negative truth-membership values, $\overrightarrow{B_{2}^{N}}\left(u_{1}, v_{1}\right), \overrightarrow{B_{2}^{N}}\left(u_{2}, v_{2}\right), \ldots$, $\overrightarrow{B_{2}^{N}}\left(u_{l}, v_{l}\right)$ be the maximum negative indeterminacy-membership values, $\overrightarrow{B_{3}^{N}}\left(u_{1}, v_{1}\right)$, $\overrightarrow{B_{3}^{N}}\left(u_{2}, v_{2}\right), \ldots, \overrightarrow{B_{3}^{N}}\left(u_{l}, v_{l}\right)$ be the minimum negative false-membership values, of edges of the paths $\overrightarrow{\mathcal{P}}_{x_{1}, w}^{m}, \overrightarrow{\mathcal{P}}_{x_{2}, w}^{m}, \ldots, \overrightarrow{\mathcal{P}}_{x_{l}, w}^{m}$, respectively. The $m$-step prey $w \in X$ is strong prey if

$$
\begin{array}{lll}
\overrightarrow{B_{1}^{P}}\left(u_{i}, v_{i}\right)>0.5, & \overrightarrow{B_{2}^{P}}\left(u_{i}, v_{i}\right)>0.5, & \overrightarrow{B_{3}^{P}}\left(u_{i}, v_{i}\right)<0.5, \\
\overrightarrow{B_{1}^{N}}\left(u_{i}, v_{i}\right)<0.5, & \overrightarrow{B_{2}^{N}}\left(u_{i}, v_{i}\right)<0.5, & \overrightarrow{B_{3}^{N}}\left(u_{i}, v_{i}\right)<0.5, \text { for all } i=1,2, \ldots, l .
\end{array}
$$

The strength of the prey $w$ can be measured by the mapping $S: X \rightarrow[0,1]$, such that:

$$
\begin{aligned}
S(w) & =\frac{1}{l}\left\{\sum_{i=1}^{l}\left[\overrightarrow{B_{1}^{P}}\left(u_{i}, v_{i}\right)\right]+\sum_{i=1}^{l}\left[\overrightarrow{B_{2}^{P}}\left(u_{i}, v_{i}\right)\right]+\sum_{i=1}^{l}\left[\overrightarrow{B_{3}^{P}}\left(u_{i}, v_{i}\right)\right]\right. \\
& \left.-\sum_{i=1}^{l}\left[\overrightarrow{B_{1}^{N}}\left(u_{i}, v_{i}\right)\right]-\sum_{i=1}^{l}\left[\overrightarrow{B_{2}^{N}}\left(u_{i}, v_{i}\right)\right]-\sum_{i=1}^{l}\left[\overrightarrow{B_{3}^{N}}\left(u_{i}, v_{i}\right)\right]\right\} .
\end{aligned}
$$

Example 2.25. Consider bipolar neutrosophic digraph $\vec{G}=(A, \vec{B})$ as shown in Fig. 7 , the strength of the prey $b$ is equal to

$$
\frac{[0.2+0.2]+[0.2+0.3]+[0.5+0.3]-[-0.3-0.2]-[-0.3-0.5]-[-0.3-0.2]}{2}=1.75
$$

Hence, $b$ is strong 2 -step prey.

Theorem 2.26. If a prey $w$ of $\vec{G}=(A, \vec{B})$ is strong, then the strength of $w$, $S(w)>0.5$.

PRoof. Let $\vec{G}=(A, \vec{B})$ be a bipolar neutrosophic digraph. Let $w$ be a common vertex of $m$-step out-neighbourhoods of vertices $x_{1}, x_{2}, \ldots, x_{l}$, i.e., there exists the paths $\overrightarrow{\mathcal{P}}_{x_{1}, w}^{m}, \overrightarrow{\mathcal{P}}_{x_{2}, w}^{m}, \ldots, \overrightarrow{\mathcal{P}}_{x_{l}, w}^{m}$, in $\vec{G}$. Also, let $\overrightarrow{B_{1}^{P}}\left(u_{1}, v_{1}\right), \overrightarrow{B_{1}^{P}}\left(u_{2}, v_{2}\right), \ldots$, $\overrightarrow{B_{1}^{P}}\left(u_{l}, v_{l}\right)$ be the minimum positive truth-membership values, $\overrightarrow{B_{2}^{P}}\left(u_{1}, v_{1}\right), \overrightarrow{B_{2}^{P}}\left(u_{2}, v_{2}\right)$, $\ldots, \overrightarrow{B_{2}^{P}}\left(u_{l}, v_{l}\right)$ be the minimum positive indeterminacy-membership values, $\overrightarrow{B_{3}^{P}}\left(u_{1}\right.$, $\left.v_{1}\right), \overrightarrow{B_{3}^{P}}\left(u_{2}, v_{2}\right), \ldots, \overrightarrow{B_{3}^{P}}\left(u_{l}, v_{l}\right)$ be the maximum positive false-membership values, 
$\overrightarrow{B_{1}^{N}}\left(u_{1}, v_{1}\right), \overrightarrow{B_{1}^{N}}\left(u_{2}, v_{2}\right), \ldots, \overrightarrow{B_{1}^{N}}\left(u_{l}, v_{l}\right)$ be the maximum negative truth-membership values, $\overrightarrow{B_{2}^{N}}\left(u_{1}, v_{1}\right), \overrightarrow{B_{2}^{N}}\left(u_{2}, v_{2}\right), \ldots, \overrightarrow{B_{2}^{N}}\left(u_{l}, v_{l}\right)$ be the maximum negative indeterminacymembership values, $\overrightarrow{B_{3}^{N}}\left(u_{1}, v_{1}\right), \overrightarrow{B_{3}^{N}}\left(u_{2}, v_{2}\right), \ldots, \overrightarrow{B_{3}^{N}}\left(u_{l}, v_{l}\right)$ be the minimum negative false-membership values, of edges of the paths $\overrightarrow{\mathcal{P}}_{x_{1}, w}^{m}, \overrightarrow{\mathcal{P}}_{x_{2}, w}^{m}, \ldots, \overrightarrow{\mathcal{P}}_{x_{l}, w}^{m}$, respectively.

If $w$ is strong, each edge $\left(u_{i}, v_{i}\right), i=1,2, \ldots, l$ is strong. So,

$$
\begin{array}{lll}
\overrightarrow{B_{1}^{P}}\left(u_{i}, v_{i}\right)>0.5, & \overrightarrow{B_{2}^{P}}\left(u_{i}, v_{i}\right)>0.5, & \overrightarrow{B_{3}^{P}}\left(u_{i}, v_{i}\right)<0.5, \\
\overrightarrow{B_{1}^{N}}\left(u_{i}, v_{i}\right)<0.5, & \overrightarrow{B_{2}^{N}}\left(u_{i}, v_{i}\right)<0.5, & \overrightarrow{B_{3}^{N}}\left(u_{i}, v_{i}\right)<0.5, \text { for all } i=1,2, \ldots, l .
\end{array}
$$

Now,

$$
S(w)>\frac{0.5+0.5+\ldots(l \text { times })+0.5}{l}>0.5 .
$$

This proves the result.

Remark: The converse of the above theorem is not true, i.e. if $S(w)>0.5$, then all preys may not be strong. This can be explained as:

Let $S(w)>0.5$ for a prey $w$ in $\vec{G}$. So,

$$
\begin{aligned}
S(w) & =\frac{1}{l}\left\{\sum_{i=1}^{l}\left[\overrightarrow{B_{1}^{P}}\left(u_{i}, v_{i}\right)\right]+\sum_{i=1}^{l}\left[\overrightarrow{B_{2}^{P}}\left(u_{i}, v_{i}\right)\right]+\sum_{i=1}^{l}\left[\overrightarrow{B_{3}^{P}}\left(u_{i}, v_{i}\right)\right]\right. \\
& \left.-\sum_{i=1}^{l}\left[\overrightarrow{B_{1}^{N}}\left(u_{i}, v_{i}\right)\right]-\sum_{i=1}^{l}\left[\overrightarrow{B_{2}^{N}}\left(u_{i}, v_{i}\right)\right]-\sum_{i=1}^{l}\left[\overrightarrow{B_{3}^{N}}\left(u_{i}, v_{i}\right)\right]\right\} .
\end{aligned}
$$

Hence,

$$
\begin{aligned}
& \left\{\sum_{i=1}^{l}\left[\overrightarrow{B_{1}^{P}}\left(u_{i}, v_{i}\right)\right]+\sum_{i=1}^{l}\left[\overrightarrow{B_{2}^{P}}\left(u_{i}, v_{i}\right)\right]+\sum_{i=1}^{l}\left[\overrightarrow{B_{3}^{P}}\left(u_{i}, v_{i}\right)\right]\right. \\
& \left.\quad-\sum_{i=1}^{l}\left[\overrightarrow{B_{1}^{N}}\left(u_{i}, v_{i}\right)\right]-\sum_{i=1}^{l}\left[\overrightarrow{B_{2}^{N}}\left(u_{i}, v_{i}\right)\right]-\sum_{i=1}^{l}\left[\overrightarrow{B_{3}^{N}}\left(u_{i}, v_{i}\right)\right]\right\}>\frac{l}{2} .
\end{aligned}
$$

This result does not necessarily imply that

$$
\begin{array}{lll}
\overrightarrow{B_{1}^{P}}\left(u_{i}, v_{i}\right)>0.5, & \overrightarrow{B_{2}^{P}}\left(u_{i}, v_{i}\right)>0.5, & \overrightarrow{B_{3}^{P}}\left(u_{i}, v_{i}\right)<0.5, \\
\overrightarrow{B_{1}^{N}}\left(u_{i}, v_{i}\right)<0.5, & \overrightarrow{B_{2}^{N}}\left(u_{i}, v_{i}\right)<0.5, & \overrightarrow{B_{3}^{N}}\left(u_{i}, v_{i}\right)<0.5, \text { for all } i=1,2, \ldots, l .
\end{array}
$$

Since, all edges of the directed paths $\overrightarrow{\mathcal{P}}_{x_{1}, w}^{m}, \overrightarrow{\mathcal{P}}_{x_{2}, w}^{m}, \ldots, \overrightarrow{\mathcal{P}}_{x_{l}, w}^{m}$, are not strong. So, the converse of the above statement is not true i.e., if $S(w)>0.5$, the prey $w$ of $\vec{G}$ may not be strong. 
Theorem 2.27. If all preys of $\vec{G}=(A, \vec{B})$ are strong, then all edges of $\mathcal{C}_{m}(\vec{G})=$ $(A, B)$ are strong.

PROOF. Let $\vec{G}=(A, \vec{B})$ be a bipolar neutrosophic digraph and all preys of it are strong. Let $\mathcal{C}_{m}(\vec{G})=(A, B)$, where,

$$
\begin{aligned}
t_{B}^{P}(x, y) & =\left[t_{A}^{P}(x) \wedge t_{A}^{P}(z)\right] h_{1}\left(\mathcal{N}_{m}^{+}(x) \cap \mathcal{N}_{m}^{+}(y)\right), \\
i_{B}^{P}(x, y) & =\left[i_{A}^{P}(x) \wedge i_{A}^{P}(y)\right] h_{2}\left(\mathcal{N}_{m}^{+}(x) \cap \mathcal{N}_{m}^{+}(y)\right), \\
f_{B}^{P}(x, y) & =\left[f_{A}^{P}(x) \vee f_{A}^{P}(y)\right] h_{3}\left(\mathcal{N}_{m}^{+}(x) \cap \mathcal{N}_{m}^{+}(y)\right), \\
t_{B}^{N}(x, y) & =\left[t_{A}^{N}(x) \vee t_{A}^{N}(z)\right] h_{4}\left(\mathcal{N}_{m}^{+}(x) \cap \mathcal{N}_{m}^{+}(y)\right), \\
i_{B}^{N}(x, y) & =\left[i_{A}^{N}(x) \vee i_{A}^{N}(y)\right] h_{5}\left(\mathcal{N}_{m}^{+}(x) \cap \mathcal{N}_{m}^{+}(y)\right), \\
f_{B}^{N}(x, y) & =\left[f_{A}^{N}(x) \wedge f_{A}^{N}(y)\right] h_{6}\left(\mathcal{N}_{m}^{+}(x) \cap \mathcal{N}_{m}^{+}(y)\right),
\end{aligned}
$$

for all edges $(x, y)$ in $\mathcal{C}_{m}(\vec{G})=(A, B)$. Then there arises two cases:

Case 1.: Let $\mathcal{N}_{m}^{+}(x) \cap \mathcal{N}_{m}^{+}(y)$ be null set. Then there does not exits any edge between $x$ and $y$ in $\mathcal{C}_{m}(\vec{G})$.

Case 2.: Let $\mathcal{N}_{m}^{+}(x) \cap \mathcal{N}_{m}^{+}(y)$ be non-empty. Now, clearly

$h_{1}\left(\mathcal{N}_{m}^{+}(x) \cap \mathcal{N}_{m}^{+}(y)\right)>0.5, \quad h_{2}\left(\mathcal{N}_{m}^{+}(x) \cap \mathcal{N}_{m}^{+}(y)\right)>0.5, \quad h_{3}\left(\mathcal{N}_{m}^{+}(x) \cap \mathcal{N}_{m}^{+}(y)\right)<0.5$, $h_{4}\left(\mathcal{N}_{m}^{+}(x) \cap \mathcal{N}_{m}^{+}(y)\right)<0.5, \quad h_{5}\left(\mathcal{N}_{m}^{+}(x) \cap \mathcal{N}_{m}^{+}(y)\right)<0.5, \quad h_{6}\left(\mathcal{N}_{m}^{+}(x) \cap \mathcal{N}_{m}^{+}(y)\right)<0.5$,

in $\vec{G}$ as all preys are strong. So, the edge $(x, y), x, y \in X$ in $\mathcal{C}_{m}(\vec{G})$ have the memberships values

$$
\begin{aligned}
t_{B}^{P}(x, y) & =\left[t_{A}^{P}(x) \wedge t_{A}^{P}(z)\right] h_{1}\left(\mathcal{N}_{m}^{+}(x) \cap \mathcal{N}_{m}^{+}(y)\right), \\
i_{B}^{P}(x, y) & =\left[i_{A}^{P}(x) \wedge i_{A}^{P}(y)\right] h_{2}\left(\mathcal{N}_{m}^{+}(x) \cap \mathcal{N}_{m}^{+}(y)\right), \\
f_{B}^{P}(x, y) & =\left[f_{A}^{P}(x) \vee f_{A}^{P}(y)\right] h_{3}\left(\mathcal{N}_{m}^{+}(x) \cap \mathcal{N}_{m}^{+}(y)\right), \\
t_{B}^{N}(x, y) & =\left[t_{A}^{N}(x) \vee t_{A}^{N}(z)\right] h_{4}\left(\mathcal{N}_{m}^{+}(x) \cap \mathcal{N}_{m}^{+}(y)\right), \\
i_{B}^{N}(x, y) & =\left[i_{A}^{N}(x) \vee i_{A}^{N}(y)\right] h_{5}\left(\mathcal{N}_{m}^{+}(x) \cap \mathcal{N}_{m}^{+}(y)\right), \\
f_{B}^{N}(x, y) & =\left[f_{A}^{N}(x) \wedge f_{A}^{N}(y)\right] h_{6}\left(\mathcal{N}_{m}^{+}(x) \cap \mathcal{N}_{m}^{+}(y)\right),
\end{aligned}
$$

and hence, all the edges are strong.

A relation is established between $m$-step bipolar neutrosophic competition graph of a bipolar neutrosophic digraph and bipolar neutrosophic competition graph of $m$-step bipolar neutrosophic digraph.

Theorem 2.28. If $\vec{G}$ is a bipolar neutrosophic digraph and $\overrightarrow{G_{m}}$ is the $m$-step bipolar neutrosophic digraph of $\vec{G}$, then $\mathcal{C}\left(\vec{G}_{m}\right)=\mathcal{C}_{m}(\vec{G})$.

PRoof. Let $\vec{G}=(A, \vec{B})$ be a bipolar neutrosophic digraph and $\overrightarrow{G_{m}}=(A, \vec{J})$ is the $m$-step bipolar neutrosophic digraph of $\vec{G}$. Also, let $\mathcal{C}\left(\vec{G}_{m}\right)=(A, J)$ and $\mathcal{C}_{m}(\vec{G})=(A, B)$. It can be easily observed that bipolar neutrosophic vertex sets of these graphs are same. So, we have to show that the bipolar neutrosophic edge 
sets of $\mathcal{C}\left(\vec{G}_{m}\right)$ and $\mathcal{C}_{m}(\vec{G})$ are equal.

Let $(x, y)$ be an edge in $\mathcal{C}\left(\vec{G}_{m}\right)$. So, there exists bipolar neutrosophic directed edges $\overrightarrow{\left(x, a_{1}\right)}, \overrightarrow{\left(y, a_{1}\right)} ; \overrightarrow{\left(x, a_{2}\right)}, \overrightarrow{\left(y, a_{2}\right)} ; \ldots ; \overrightarrow{\left(x, a_{l}\right)}, \overrightarrow{\left(y, a_{l}\right)}$, for some positive integer $l$ in $\vec{G}_{m}$. Now, in $\vec{G}_{m}$,

$$
\mathcal{N}^{+}(x) \cap \mathcal{N}^{+}(y)=\left\{\left(a_{i}, s_{i}^{P}, q_{i}^{P}, r_{i}^{P}, s_{i}^{N}, q_{i}^{N}, r_{i}^{N}\right) \mid i=1,2, \ldots, l\right\},
$$

where,

$$
\begin{aligned}
s_{i}^{P}=\vec{J}\left(x, a_{i}\right) \wedge \vec{J}\left(y, a_{i}\right), & s_{i}^{N}=\vec{J}\left(x, a_{i}\right) \vee \vec{J}\left(y, a_{i}\right), \\
q_{i}^{P}=\vec{J}\left(x, a_{i}\right) \wedge \vec{J}\left(y, a_{i}\right), & q_{i}^{N}=\vec{J}\left(x, a_{i}\right) \vee \vec{J}\left(y, a_{i}\right), \\
r_{i}^{P}=\vec{J}\left(x, a_{i}\right) \vee \vec{J}\left(y, a_{i}\right), & r_{i}^{N}=\vec{J}\left(x, a_{i}\right) \wedge \vec{J}\left(y, a_{i}\right) .
\end{aligned}
$$

Let

$$
\begin{array}{ll}
S^{P}=\max \left\{s_{i}^{P} \mid i=1,2, \ldots, l\right\}, & S^{N}=\min \left\{s_{i}^{N} \mid i=1,2, \ldots, l\right\}, \\
Q^{P}=\max \left\{q_{i}^{P} \mid i=1,2, \ldots, l\right\}, & Q^{N}=\min \left\{q_{i}^{N} \mid i=1,2, \ldots, l\right\}, \\
R^{P}=\min \left\{r_{i}^{P} \mid i=1,2, \ldots, l\right\}, & R^{N}=\max \left\{r_{i}^{N} \mid i=1,2, \ldots, l\right\} .
\end{array}
$$

Hence,

$$
\begin{aligned}
t_{J}^{P}(x, y) & =\left(t_{A}^{P}(x) \wedge t_{A}^{P}(y)\right) h_{1}\left(\mathcal{N}^{+}(x) \cap \mathcal{N}^{+}(y)\right)=S^{P} \times t_{A}^{P}(x) \wedge t_{A}^{P}(y), \\
i_{J}^{P}(x, y) & =\left(i_{A}^{P}(x) \wedge i_{A}^{P}(y)\right) h_{2}\left(\mathcal{N}^{+}(x) \cap \mathcal{N}^{+}(y)\right)=Q^{P} \times i_{A}^{P}(x) \wedge i_{A}^{P}(y), \\
f_{J}^{P}(x, y) & =\left(f_{A}^{P}(x) \vee f_{A}^{P}(y)\right) h_{3}\left(\mathcal{N}^{+}(x) \cap \mathcal{N}^{+}(y)\right)=R^{P} \times f_{A}^{P}(x) \vee f_{A}^{P}(y), \\
t_{J}^{N}(x, y) & =\left(t_{A}^{N}(x) \vee t_{A}^{N}(y)\right) h_{4}\left(\mathcal{N}^{+}(x) \cap \mathcal{N}^{+}(y)\right)=S^{N} \times t_{A}^{N}(x) \vee t_{A}^{N}(y), \\
i_{J}^{N}(x, y) & =\left(i_{A}^{N}(x) \vee i_{A}^{N}(y)\right) h_{5}\left(\mathcal{N}^{+}(x) \cap \mathcal{N}^{+}(y)\right)=Q^{N} \times i_{A}^{N}(x) \vee i_{A}^{N}(y), \\
f_{J}^{N}(x, y) & =\left(f_{A}^{N}(x) \wedge f_{A}^{N}(y)\right) h_{6}\left(\mathcal{N}^{+}(x) \cap \mathcal{N}^{+}(y)\right)=R^{N} \times f_{A}^{N}(x) \wedge f_{A}^{N}(y) .
\end{aligned}
$$

An edge $\overrightarrow{\left(x, a_{i}\right)}$ exists in $\vec{G}_{m}$ that implies there exists a bipolar neutrosophic directed path from $x$ to $a_{i}$ of length $m, \overrightarrow{\mathcal{P}}_{x, a_{i}}^{m}$ in $\vec{G}$ and

$$
\begin{aligned}
& \vec{J}_{1}^{P}\left(x, a_{i}\right)=\min \left\{\vec{B}_{1}^{P}(u, v) \mid(u, v) \text { is an edge in } \overrightarrow{\mathcal{P}}_{x, a_{i}}^{m}\right\}, \\
& \vec{J}_{2}^{P}\left(x, a_{i}\right)=\min \left\{\vec{B}_{2}^{P}(u, v) \mid(u, v) \text { is an edge in } \overrightarrow{\mathcal{P}}_{x, a_{i}}^{m}\right\}, \\
& \vec{J}_{3}^{P}\left(x, a_{i}\right)=\max \left\{\vec{B}_{3}^{P}(u, v) \mid(u, v) \text { is an edge in } \overrightarrow{\mathcal{P}}_{x, a_{i}}^{m}\right\}, \\
& \vec{J}_{1}^{N}\left(x, a_{i}\right)=\max \left\{\vec{B}_{1}^{N}(u, v) \mid(u, v) \text { is an edge in } \overrightarrow{\mathcal{P}}_{x, a_{i}}^{m}\right\}, \\
& \vec{J}_{2}^{N}\left(x, a_{i}\right)=\max \left\{\vec{B}_{2}^{N}(u, v) \mid(u, v) \text { is an edge in } \overrightarrow{\mathcal{P}}_{x, a_{i}}^{m}\right\}, \\
& \vec{J}_{3}^{N}\left(x, a_{i}\right)=\min \left\{\vec{B}_{3}^{N}(u, v) \mid(u, v) \text { is an edge in } \overrightarrow{\mathcal{P}}_{x, a_{i}}^{m}\right\}
\end{aligned}
$$


Thus, the edge $(x, y)$ is also available in $\mathcal{C}_{m}(\vec{G})$. Also,

$$
\begin{array}{ll}
h_{1}\left(\mathcal{N}_{m}^{+}(x) \cap \mathcal{N}_{m}^{+}(y)\right)=S^{P}, & h_{4}\left(\mathcal{N}_{m}^{+}(x) \cap \mathcal{N}_{m}^{+}(y)\right)=S^{N}, \\
h_{2}\left(\mathcal{N}_{m}^{+}(x) \cap \mathcal{N}_{m}^{+}(y)\right)=Q^{P}, & h_{5}\left(\mathcal{N}_{m}^{+}(x) \cap \mathcal{N}_{m}^{+}(y)\right)=Q^{N}, \\
h_{3}\left(\mathcal{N}_{m}^{+}(x) \cap \mathcal{N}_{m}^{+}(y)\right)=R^{P}, & h_{6}\left(\mathcal{N}_{m}^{+}(x) \cap \mathcal{N}_{m}^{+}(y)\right)=R^{N},
\end{array}
$$

in $\vec{G}$. Hence, finally

$$
\begin{aligned}
t_{B}^{P}(x, y) & =\left[t_{A}^{P}(x) \wedge t_{A}^{P}(z)\right] h_{1}\left(\mathcal{N}_{m}^{+}(x) \cap \mathcal{N}_{m}^{+}(y)\right)=S^{P} \times t_{A}^{P}(x) \wedge t_{A}^{P}(z), \\
i_{B}^{P}(x, y) & =\left[i_{A}^{P}(x) \wedge i_{A}^{P}(y)\right] h_{2}\left(\mathcal{N}_{m}^{+}(x) \cap \mathcal{N}_{m}^{+}(y)\right)=Q^{P} \times i_{A}^{P}(x) \wedge i_{A}^{P}(y), \\
f_{B}^{P}(x, y) & =\left[f_{A}^{P}(x) \vee f_{A}^{P}(y)\right] h_{3}\left(\mathcal{N}_{m}^{+}(x) \cap \mathcal{N}_{m}^{+}(y)\right)=R^{P} \times f_{A}^{P}(x) \vee f_{A}^{P}(y), \\
t_{B}^{N}(x, y) & =\left[t_{A}^{N}(x) \vee t_{A}^{N}(z)\right] h_{4}\left(\mathcal{N}_{m}^{+}(x) \cap \mathcal{N}_{m}^{+}(y)\right)=S^{N} \times t_{A}^{N}(x) \vee t_{A}^{N}(z), \\
i_{B}^{N}(x, y) & =\left[i_{A}^{N}(x) \vee i_{A}^{N}(y)\right] h_{5}\left(\mathcal{N}_{m}^{+}(x) \cap \mathcal{N}_{m}^{+}(y)\right)=Q^{N} \times i_{A}^{N}(x) \vee i_{A}^{N}(y), \\
f_{B}^{N}(x, y) & =\left[f_{A}^{N}(x) \wedge f_{A}^{N}(y)\right] h_{6}\left(\mathcal{N}_{m}^{+}(x) \cap \mathcal{N}_{m}^{+}(y)\right)=R^{N} \times f_{A}^{N}(x) \wedge f_{A}^{N}(y) .
\end{aligned}
$$

This proves that there exists an edge in $\mathcal{C}_{m}(\vec{G})$ for every edge in $\mathcal{C}\left(\vec{G}_{m}\right)$. Similarly, for every edge in $\mathcal{C}_{m}(\vec{G})$ there exists an edge in $\mathcal{C}\left(\vec{G}_{m}\right)$. This proves that $\mathcal{C}\left(\vec{G}_{m}\right)=$ $\mathcal{C}_{m}(\vec{G})$.

Theorem 2.29. Let $\vec{G}=(A, \vec{B})$ be a bipolar neutrosophic digraph. If $m>|X|$ then $\mathcal{C}_{m}(\vec{G})=(A, B)$ has no edge.

PROOF. Let $\vec{G}=(A, \vec{B})$ be a bipolar neutrosophic digraph and $\mathcal{C}_{m}(\vec{G})=(A, B)$ be the corresponding $m$-step bipolar neutrosophic competition graph, where,

$$
\begin{aligned}
t_{B}^{P}(x, y) & =\left[t_{A}^{P}(x) \wedge t_{A}^{P}(z)\right] h_{1}\left(\mathcal{N}_{m}^{+}(x) \cap \mathcal{N}_{m}^{+}(y)\right), \\
i_{B}^{P}(x, y) & =\left[i_{A}^{P}(x) \wedge i_{A}^{P}(y)\right] h_{2}\left(\mathcal{N}_{m}^{+}(x) \cap \mathcal{N}_{m}^{+}(y)\right), \\
f_{B}^{P}(x, y) & =\left[f_{A}^{P}(x) \vee f_{A}^{P}(y)\right] h_{3}\left(\mathcal{N}_{m}^{+}(x) \cap \mathcal{N}_{m}^{+}(y)\right), \\
t_{B}^{N}(x, y) & =\left[t_{A}^{N}(x) \vee t_{A}^{N}(z)\right] h_{4}\left(\mathcal{N}_{m}^{+}(x) \cap \mathcal{N}_{m}^{+}(y)\right), \\
i_{B}^{N}(x, y) & =\left[i_{A}^{N}(x) \vee i_{A}^{N}(y)\right] h_{5}\left(\mathcal{N}_{m}^{+}(x) \cap \mathcal{N}_{m}^{+}(y)\right), \\
f_{B}^{N}(x, y) & =\left[f_{A}^{N}(x) \wedge f_{A}^{N}(y)\right] h_{6}\left(\mathcal{N}_{m}^{+}(x) \cap \mathcal{N}_{m}^{+}(y)\right),
\end{aligned}
$$

for all edges $(x, y)$ in $\mathcal{C}_{m}(\vec{G})$.

If $m>|X|$, there does not exist any directed bipolar neutrosophic path of length $m$ in $\vec{G}$. So, $\mathcal{N}_{m}^{+}(x) \cap \mathcal{N}_{m}^{+}(y)$ ia an empty set. Hence, there does not exist any edge in $\mathcal{C}_{m}(\vec{G})$.

Now, $m$-step bipolar neutrosophic neighbouhood graphs are defines below.

Definition 2.30. The bipolar neutrosophic m-step out-neighbourhood of vertex $x$ of a bipolar neutrosophic digraph $\vec{G}=(A, \vec{B})$ is bipolar neutrosophic set 


$$
\mathcal{N}_{m}(x)=\left(X_{x}, t_{x}^{P}, i_{x}^{P}, f_{x}^{P}, t_{x}^{N}, i_{x}^{N}, f_{x}^{N}\right), \quad \text { where }
$$

$X_{x}=\{y \mid$ there exists a directed bipolar neutrosophic path of length $m$ from $x$ to $\left.y, \mathcal{P}_{x, y}^{m}\right\}, t_{x}^{P}: X_{x} \rightarrow[0,1], i_{x}^{P}: X_{x} \rightarrow[0,1], f_{x}^{P}: X_{x} \rightarrow[0,1], t_{x}^{N}: X_{x} \rightarrow[-1$, $0], i_{x}^{N}: X_{x} \rightarrow[-1,0], f_{x}^{N}: X_{x} \rightarrow[-1,0]$, are defined by $t_{x}^{P}=\min \left\{t^{P}\left(x_{1}\right.\right.$, $\left.x_{2}\right),\left(x_{1}, x_{2}\right)$ is an edge of $\left.\mathcal{P}_{x, y}^{m}\right\}, i_{x}^{P}=\min \left\{i^{P}\left(x_{1}, x_{2}\right),\left(x_{1}, x_{2}\right)\right.$ is an edge of $\left.\mathcal{P}_{x, y}^{m}\right\}, f_{x}^{P}=\max \left\{f^{P}\left(x_{1}, x_{2}\right),\left(x_{1}, x_{2}\right)\right.$ is an edge of $\left.\mathcal{P}_{x, y}^{m}\right\}, t_{x}^{N}=\max \left\{t^{N}\left(x_{1}, x_{2}\right)\right.$, $\left(x_{1}, x_{2}\right)$ is an edge of $\left.\mathcal{P}_{x, y}^{m}\right\}, i_{x}^{N}=\max \left\{i^{N}\left(x_{1}, x_{2}\right),\left(x_{1}, x_{2}\right)\right.$ is an edge of $\left.\mathcal{P}_{x, y}^{m}\right\}$, $f_{x}^{N}=\min \left\{f^{N}\left(x_{1}, x_{2}\right),\left(x_{1}, x_{2}\right)\right.$ is an edge of $\left.\mathcal{P}_{x, y}^{m}\right\}$, respectively.

Definition 2.31. Suppose $G=(A, B)$ is a bipolar neutrosophic graph. Then m-step bipolar neutrosophic neighbouhood graph $\mathcal{N}_{m}(G)$ is defined by $\mathcal{N}_{m}(G)=(A$, $\dot{B})$ where $A=\left(A_{1}^{P}, A_{2}^{P}, A_{3}^{P}, A_{1}^{N}, A_{2}^{N}, A_{3}^{N}\right), \dot{B}=\left(\dot{B}_{1}^{P}, \dot{B}_{2}^{P}, \dot{B}_{3}^{P}, \dot{B}_{1}^{N}, \dot{B}_{2}^{N}, \dot{B}_{3}^{N}\right)$, $\dot{B}_{1}^{P}: X \times X \rightarrow[0,1], \dot{B}_{2}^{P}: X \times X \rightarrow[0,1], \dot{B}_{3}^{P}: X \times X \rightarrow[0,1], \hat{B}_{1}^{N}: X \times X \rightarrow[-1$, $0], \dot{B}_{2}^{N}: X \times X \rightarrow[-1,0]$, and $\dot{B}_{3}^{N}: X \times X \rightarrow[-1,0]$ are such that:

$$
\begin{aligned}
& \dot{B}_{1}^{P}(x, y)=A_{1}^{P}(x) \wedge A_{1}^{P}(y) h_{1}\left(\mathcal{N}_{m}(x) \cap \mathcal{N}_{m}(y)\right), \\
& \dot{B}_{2}^{P}(x, y)=A_{2}^{P}(x) \wedge A_{2}^{P}(y) h_{2}\left(\mathcal{N}_{m}(x) \cap \mathcal{N}_{m}(y)\right), \\
& \dot{B}_{3}^{P}(x, y)=A_{3}^{P}(x) \vee A_{3}^{P}(y) h_{3}\left(\mathcal{N}_{m}(x) \cap \mathcal{N}_{m}(y)\right), \\
& \dot{B}_{1}^{N}(x, y)=A_{1}^{N}(x) \vee A_{1}^{N}(y) h_{4}\left(\mathcal{N}_{m}(x) \cap \mathcal{N}_{m}(y)\right), \\
& \dot{B}_{2}^{N}(x, y)=A_{2}^{N}(x) \vee A_{2}^{N}(y) h_{5}\left(\mathcal{N}_{m}(x) \cap \mathcal{N}_{m}(y)\right), \\
& \dot{B}_{3}^{N}(x, y)=A_{3}^{N}(x) \wedge A_{3}^{N}(y) h_{6}\left(\mathcal{N}_{m}(x) \cap \mathcal{N}_{m}(y)\right), \quad \text { respectively. }
\end{aligned}
$$

Definition 2.32. [?]Consider a bipolar neutrosophic graph $G=(A, B)$, where $A=\left(A_{1}^{P}, A_{2}^{P}, A_{3}^{P}, A_{1}^{N}, A_{2}^{N}, A_{3}^{N}\right)$, and $B=\left(B_{1}^{P}, B_{2}^{P}, B_{3}^{P}, B_{1}^{N}, B_{2}^{N}, B_{3}^{N}\right)$ then, an edge $(x, y), x, y \in X$ is called independent strong if

$$
\begin{array}{ll}
\frac{1}{2}\left[A_{1}^{P}(x) \wedge A_{1}^{P}(y)\right]<B_{1}^{P}(x, y), & \frac{1}{2}\left[A_{1}^{N}(x) \vee A_{1}^{N}(y)\right]>B_{1}^{N}(x, y), \\
\frac{1}{2}\left[A_{2}^{P}(x) \wedge A_{2}^{P}(y)\right]<B_{2}^{P}(x, y), & \frac{1}{2}\left[A_{2}^{N}(x) \vee A_{2}^{N}(y)\right]>B_{2}^{N}(x, y), \\
\frac{1}{2}\left[A_{3}^{P}(x) \vee A_{3}^{P}(y)\right]>B_{3}^{P}(x, y), & \frac{1}{2}\left[A_{3}^{N}(x) \wedge A_{3}^{N}(y)\right]<B_{3}^{N}(x, y) .
\end{array}
$$

Otherwise, it is called weak.

Theorem 2.33. If all the edges of bipolar neutrosophic digraph $\vec{G}=(A, \vec{B})$ are independent strong, then all the edges of $\mathcal{C}_{m}(\vec{G})$ are independent strong.

PRoof. Suppose $\vec{G}=(A, \vec{B})$ is a bipolar neutrosophic digraph and $\mathcal{C}_{m}(\vec{G})=(A$, $B)$ is corresponding $m$-step bipolar neutrosophic competition graph. Since all the 
edges of $\vec{G}$ are independent strong, then

$\begin{array}{lll}h_{1}\left(\mathcal{N}_{m}^{+}(x) \cap \mathcal{N}_{m}^{+}(y)\right)>0.5, & h_{2}\left(\mathcal{N}_{m}^{+}(x) \cap \mathcal{N}_{m}^{+}(y)\right)>0.5, & h_{3}\left(\mathcal{N}_{m}^{+}(w) \cap \mathcal{N}_{m}^{+}(z)\right)<0.5, \\ h_{4}\left(\mathcal{N}_{m}^{+}(x) \cap \mathcal{N}_{m}^{+}(y)\right)<0.5, & h_{5}\left(\mathcal{N}_{m}^{+}(x) \cap \mathcal{N}_{m}^{+}(y)\right)<0.5, & h_{6}\left(\mathbb{N}_{m}^{+}(w) \cap \mathbb{N}_{m}^{+}(z)\right)<0.5 .\end{array}$

Then,

$t_{B}^{P}(x, y)=\left(t_{A}^{P}(x) \wedge t_{A}^{P}(y)\right) h_{1}\left(\mathcal{N}_{m}^{+}(x) \cap \mathcal{N}_{m}^{+}(y)\right)$ or, $t_{B}^{P}(x, y)>0.5\left(t_{A}^{P}(x) \wedge t_{A}^{P}(y)\right)$ or, $\frac{t_{B}^{P}(x, y)}{\left(t_{A}^{P}(x) \wedge t_{A}^{P}(y)\right)}>0.5, i_{B}^{P}(x, y)=\left(i_{A}^{P}(x) \wedge i_{A}^{P}(y)\right) h_{2}\left(\mathcal{N}_{m}^{+}(x) \cap \mathcal{N}_{m}^{+}(y)\right)$ or, $i_{B}^{P}(x, y)>$ $0.5\left(i_{A}^{P}(x) \wedge i_{A}^{P}(y)\right)$ or, $\frac{i_{B}^{P}(x, y)}{\left(i_{A}^{P}(x) \wedge i_{A}^{P}(y)\right)}>0.5, f_{B}^{P}(x, y)=\left(f_{A}^{P}(x) \vee f_{A}^{P}(y)\right) h_{3}\left(\mathcal{N}_{m}^{+}(x) \cap\right.$ $\left.\mathcal{N}_{m}^{+}(y)\right)$ or, $f_{B}^{P}(x, y)<0.5\left(f_{A}^{P}(x) \vee f_{A}^{P}(y)\right)$ or, $\frac{f_{B}^{P}(x, y)}{\left(f_{A}^{P}(x) \vee f_{A}^{P}(y)\right)}<0.5, t_{B}^{N}(x, y)=$ $\left(t_{A}^{N}(x) \vee t_{A}^{N}(y)\right) h_{4}\left(\mathcal{N}_{m}^{+}(x) \cap \mathcal{N}_{m}^{+}(y)\right)$ or, $t_{B}^{N}(x, y)<0.5\left(t_{A}^{N}(x) \vee t_{A}^{N}(y)\right)$ or, $\frac{t_{B}^{N}(x, y)}{\left(t_{A}^{N}(x) \vee t_{A}^{N}(y)\right)}<$ $0.5, i_{B}^{N}(x, y)=\left(i_{A}^{N}(x) \vee i_{A}^{N}(y)\right) h_{5}\left(\mathcal{N}_{m}^{+}(x) \cap \mathcal{N}_{m}^{+}(y)\right)$ or, $i_{B}^{N}(x, y)<0.5\left(i_{A}^{N}(x) \vee\right.$ $\left.i_{A}^{N}(y)\right)$ or, $\frac{i_{B}^{N}(x, y)}{\left(i_{A}^{N}(x) \vee i_{A}^{N}(y)\right)}<0.5, f_{B}^{N}(x, y)=\left(f_{A}^{N}(x) \wedge f_{A}^{N}(y)\right) h_{6}\left(\mathcal{N}_{m}^{+}(x) \cap \mathcal{N}_{m}^{+}(y)\right)$ or, $f_{B}^{N}(x, y)<0.5\left(f_{A}^{N}(x) \wedge f_{A}^{N}(y)\right)$ or, $\frac{f_{B}^{N}(x, y)}{\left(f_{A}^{N}(x) \wedge f_{A}^{N}(y)\right)}<0.5$.

Hence, the edge $(x, y)$ is independent strong in $\mathcal{C}_{m}(\vec{G})$. Since, $(x, y)$ is taken to be arbitrary edge of $\mathcal{C}_{m}(\vec{G})$, thus all the edges of $\mathcal{C}_{m}(\vec{G})$ are independent strong.

\section{Application}

Sports are very important, every society has its own special kinds of sports. The proper end of sports is bodily health arid physical fitness. Sports and games have now come to stay in our civilization as an essential feature of human activity, and their object is not merely fun, they also instill the sprit of discipline and teamwork. Sports like cricket, hockey and foot ball are popular because of the sprit of team work which they inspire. This no doubt true. The discipline that gained in playing up sports is invaluable in later life. It makes for a life of co-operation and team work which could be used for building up a great society and a nation. Key components of sports are goals, rules, challenge, and interaction. Sports generally involve mental or physical stimulation, and often both. Many sports help develop practical skills, serve as a form of exercise, or otherwise perform an educational, simulational, or psychological role etc. Many sports require special equipment and dedicated playing fields, leading to the involvement of a community much larger than the group of players. A city or town may set aside such resources for the organization of sports leagues, like, tabletop games, board games, etc. All these types of sports are called local sports. These sports can be extended to provisional level sports. After provisional level sports there are national sports. A national sport is a sport or game that is considered to be an intrinsic part of the culture of a nation. Every nation has different sports, such as, baseball is known as national sports in the United States, cricket is in England, and hockey is in Pakistan, etc. After, national level of sports there are international level of sports. International sport is a sport in which the participants represent different countries. The most 
well-known international sports event is the Olympic Games, FIFA World Cup and the Paralympic Games.

Consider the set consisting of three countries $\left\{C_{1}, C_{2}, C_{3}\right\}$ and also consider the set of players $\{($ Abigail, 0.9, 0.8, 0.5, -0.6, -0.5, -0.2), (Alex, 0.6, 0.3, 0.4, -0.2, -0.4, -0.4),(Amelia, 0.8, 0.7, 0.2, - 0.7, -0.8, -0.5), (Agatha, 0.9, 0.8, 0.5, -0.6, $-0.5,-0.2$ ), (Angela, 0.9, 0.8, 0.5, - 0.6, -0.5, -0.2), (Belinda, 0.9, 0.8, 0.5, -0.6, $-0.5,-0.2$ ), (Ann, 0.5, 0.3, 0.5, -0.5, -0.3, -0.2), (Arlene, 0.8, 0.8, 0.9, -0.8, $-0.9,-0.8$ ), (Bella, 0.6, 0.4, 0.9, - 0.6, -0.7, -0.5), (Anne, 0.9, 0.7, 0.8, -0.8, $-0.8,-0.8$ ), (April, 0.5, 0.3, 0.5, - 0.5, - 0.3, -0.2), (Abbey, 0.5, 0.3, 0.5, - 0.5, $-0.3,-0.2)\}$, which are taking part in their local, provisional, national, and international level games, as shown in Fig. 9. The positive degree of membership $t^{P}(x)$ of each player represent the percentage of hardwork towards to achieve the success in particular game, $i^{P}(x)$ and $f^{P}(x)$ represent the indeterminacy and falsity in this percentage. The negative degree of membership $t^{N}(x)$ represents the percentage that the player faces failure in the achievement of success in a particular game, $i^{N}(x)$ and $f^{N}(x)$ represent the indeterminacy and falsity in this percentage. The positive degree of membership $t^{P}(x)$ of each directed edge between player and local, provisional, national and international level games represent the percentage of having stamina for that level of sports in international game, $i^{P}(x)$ and $f^{P}(x)$ represent the indeterminacy and falsity in this percentage. The negative degree of membership $t^{N}(x)$ of each directed edge between player and local, provisional, national and international level games represent the percentage of having no stamina for that level of sports in international game, $i^{N}(x)$ and $f^{N}(x)$ represent the indeterminacy and falsity in this percentage.

Thus, 4-step bipolar neutrosophic competition graph can be used in order to find the best results. There 4-step bipolar neutrosophic out-neighbourhoods is calculated in Table 2.

TABLE 2. 4-Step bipolar neutrosophic out-neighbourhoods

\begin{tabular}{|l|l|}
\hline$x \in X$ & $\mathcal{N}_{4}^{+}(x)$ \\
\hline Abigail & $\{($ International games, $0.2,0.2,0.6,-0.1,-0.3,-0.4)\}$ \\
Alex & $\{($ International games, $0.4,0.2,0.6,-0.1,-0.2,-0.7)\}$ \\
Amelia & $\{($ International games, $0.5,0.5,0.6,-0.2,-0.2,-0.8)\}$ \\
\hline
\end{tabular}

Therefore, $\mathcal{N}_{4}^{+}($Abigail $) \cap \mathcal{N}_{4}^{+}($Alex $)=\{($International games, 0.2, 0.2, 0.6, - 0.1, $-0.2,-0.7)\}, \mathcal{N}_{4}^{+}($Abigail $) \cap \mathcal{N}_{4}^{+}($Amelia $)=\{($International games, $0.2,0.2,0.6$, $-0.1,-0.2,-0.8)\}$, and $\mathcal{N}_{4}^{+}($Alex $) \cap \mathcal{N}_{4}^{+}($Amelia $)=\{($International games, 0.4 , $0.2,0.6,-0.1,-0.2,-0.8)\}$. Further, $h\left(\mathcal{N}_{4}^{+}(\right.$Abigail $) \cap \mathcal{N}_{4}^{+}($Alex $\left.)\right)=(0.2,0.2,0.6$, $0.2,0.2,0.6), h\left(\mathcal{N}_{4}^{+}(\right.$Abigail $) \cap \mathcal{N}_{4}^{+}($Amelia $\left.)\right)=(0.2,0.2,0.6,0.2,0.2,0.6)$, and $h\left(\mathcal{N}_{4}^{+}(\right.$Amelia $) \cap \mathcal{N}_{4}^{+}($Alex $\left.)\right)=(0.4,0.2,0.6,0.4,0.2,0.6)$. Thus, we obtain 4-step bipolar neutrosophic competition graph, as shown in Fig. 10. 


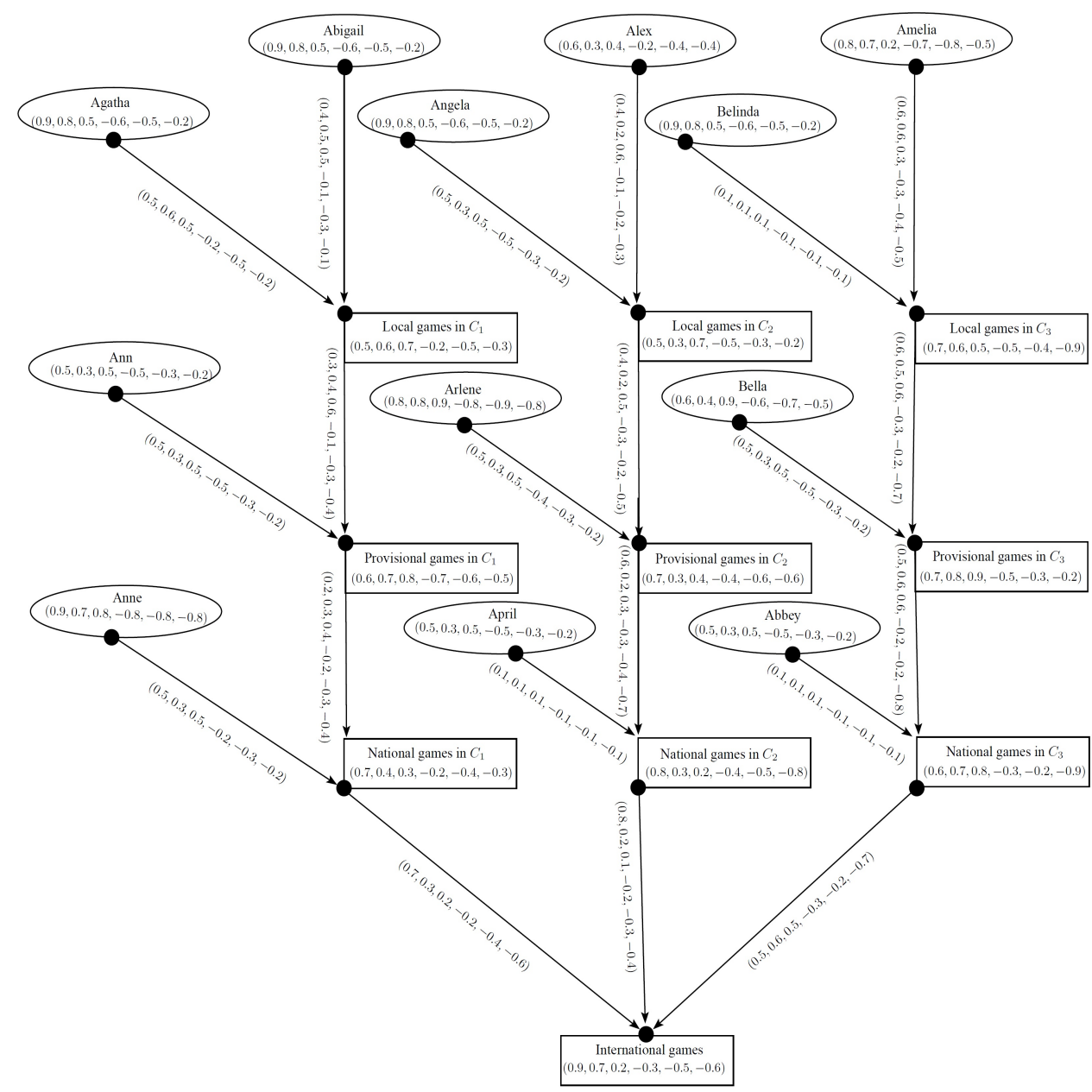

FIGURE 9. Bipolar neutrosophic digraph

TABLE 3. Strength of competition of applicants for international games

\begin{tabular}{|l|l|l|}
\hline$(x, y)$ & $T(x, y)$ & $S(x, y)$ \\
\hline (Abigail, Alex) & $(0.12,0.06,0.30,-0.04,-0.08,-0.24)$ & 1.04 \\
\hline (Abigail, Amelia) & $(0.16,0.14,0.30,-0.12,-0.10,-0.30)$ & 1 \\
\hline (Alex, Amelia) & $(0.24,0.06,0.24,-0.08,-0.08,-0.30)$ & 1.24 \\
\hline
\end{tabular}

The strength to compete the others players with respect hardwork in order to achieve success is calculated in Table 3. In Table 3, $T(x, y)$ represents the value of strength of competition between players $x$ and $y$ with respect to hardwork to 


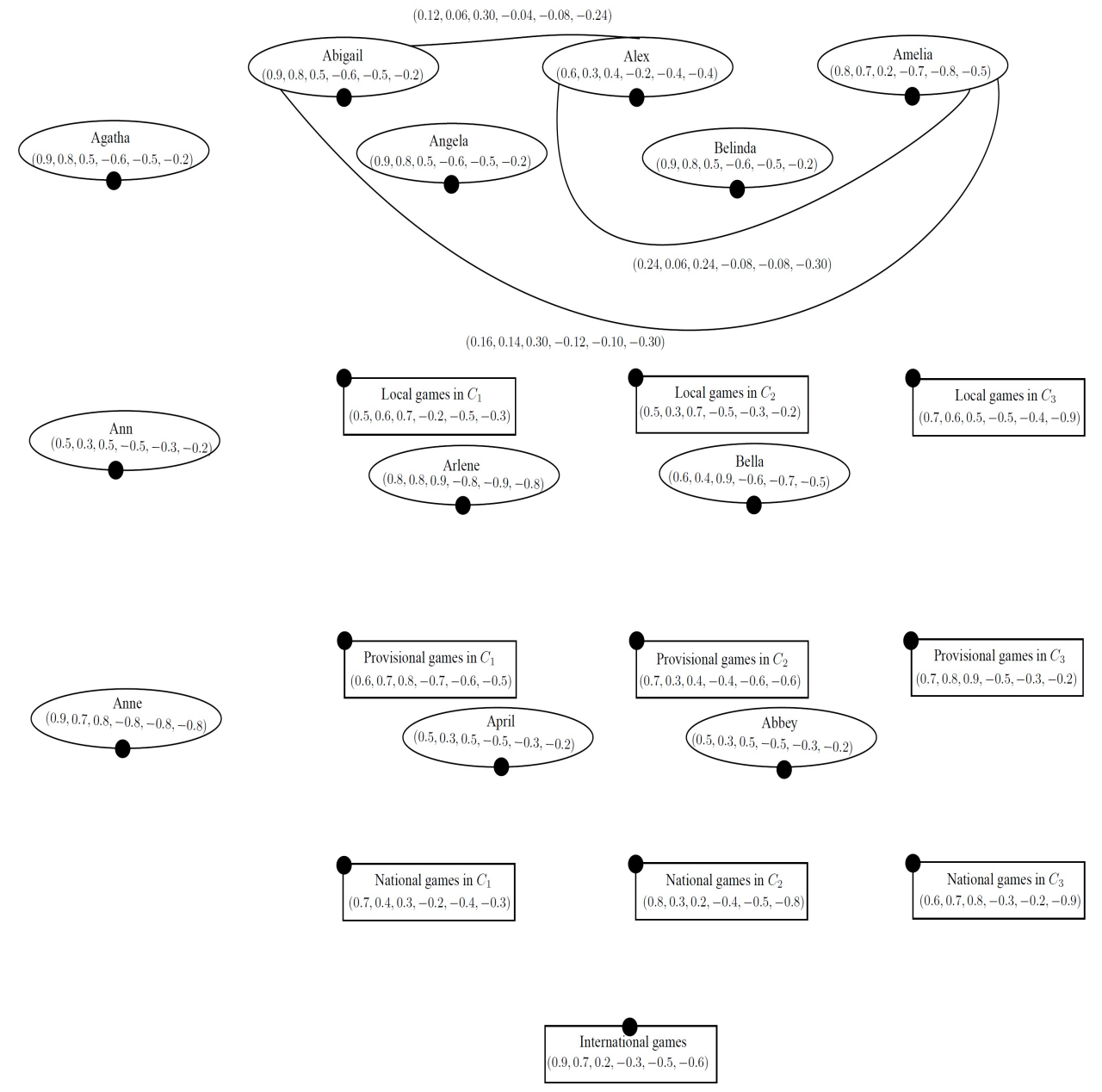

FIGURE 10. 4-Step bipolar neutrosophic competition graph

achieve the success in particular game. From Table 3, it is clear that the strength of competition between Alex and Amelia to achieve the success in particular game in international level is 1.24, while strength of competition between between Abigail and Amelia is 1, and strength of competition between between Abigail and Alex is 1.04. It is also clear from the Table 3, that Alex and Amelia are strongest contestants, as the strength of competition between them has the largest value than the other contestants.

We now elaborate this method with the help of an algorithm. 


\section{Algorithm}

Step 1.: Input the positive truth, indeterminacy and falsity-memberships values and negative truth, indeterminacy and falsity-memberships values for set of $r$ applicants.

Step 2.: If for any two distinct vertices $x_{i}$ and $x_{j}, t^{P}\left(x_{i} x_{j}\right)>0, i^{P}\left(x_{i} x_{j}\right)>0$, $f^{P}\left(x_{i} x_{j}\right)>0, t^{N}\left(x_{i} x_{j}\right)<0, i^{N}\left(x_{i} x_{j}\right)<0, f^{N}\left(x_{i} x_{j}\right)<0$, then

$\left(x_{j}, t^{P}\left(x_{i} x_{j}\right), i^{P}\left(x_{i} x_{j}\right), f^{P}\left(x_{i} x_{j}\right), t^{N}\left(x_{i} x_{j}\right), i^{N}\left(x_{i} x_{j}\right), f^{N}\left(x_{i} x_{j}\right)\right) \in \mathcal{N}_{m}^{+}\left(x_{i}\right)$.

Step 3.: Repeat step 2 for all vertices $x_{i}$ and $x_{j}$ to calculate $m$-step bipolar neutrosophic-out-neighbourhoods $\mathcal{N}_{m}^{+}\left(x_{i}\right)$.

Step 4.: Calculate $\mathcal{N}_{m}^{+}\left(x_{i}\right) \cap \mathcal{N}_{m}^{+}\left(x_{j}\right)$ for each pair of distinct vertices $x_{i}$ and $x_{j}$.

Step 5.: Calculate $h\left[\mathcal{N}_{m}^{+}\left(x_{i}\right) \cap \mathcal{N}_{m}^{+}\left(x_{j}\right)\right]$.

Step 6.: If $\mathcal{N}_{m}^{+}\left(x_{i}\right) \cap \mathcal{N}_{m}^{+}\left(x_{j}\right) \neq \emptyset$ then draw an edge $x_{i} x_{j}$.

Step 7.: Repeat step 6 for all pair of distinct vertices.

Step 8.: Assign membership values to each edge $x_{i} x_{j}$ using the conditions

$$
\begin{aligned}
& t^{P}\left(x_{i} x_{j}\right)=\left(x_{i} \wedge x_{j}\right) h_{1}\left[\mathcal{N}_{m}^{+}\left(x_{i}\right) \cap \mathcal{N}_{m}^{+}\left(x_{j}\right)\right] \quad t^{N}\left(x_{i} x_{j}\right)=\left(x_{i} \vee x_{j}\right) h_{4}\left[\mathcal{N}_{m}^{+}\left(x_{i}\right) \cap \mathcal{N}_{m}^{+}\left(x_{j}\right)\right] \\
& i^{P}\left(x_{i} x_{j}\right)=\left(x_{i} \wedge x_{j}\right) h_{2}\left[\mathcal{N}_{m}^{+}\left(x_{i}\right) \cap \mathcal{N}_{m}^{+}\left(x_{j}\right)\right] \quad i^{N}\left(x_{i} x_{j}\right)=\left(x_{i} \vee x_{j}\right) h_{5}\left[\mathcal{N}_{m}^{+}\left(x_{i}\right) \cap \mathcal{N}_{m}^{+}\left(x_{j}\right)\right] \\
& f^{P}\left(x_{i} x_{j}\right)=\left(x_{i} \vee x_{j}\right) h_{3}\left[\mathcal{N}_{m}^{+}\left(x_{i}\right) \cap \mathcal{N}_{m}^{+}\left(x_{j}\right)\right] \quad f^{N}\left(x_{i} x_{j}\right)=\left(x_{i} \wedge x_{j}\right) h_{6}\left[\mathcal{N}_{m}^{+}\left(x_{i}\right) \cap \mathcal{N}_{m}^{+}\left(x_{j}\right)\right] .
\end{aligned}
$$

Step 10.: Calculate $S(x, y)$, the strength of competition between players $x$ and $y$.

$$
\begin{gathered}
S(x, y)=t^{P}(x, y)-\left(i^{P}(x, y)+f^{P}(x, y)\right)+1+t^{N}(x, y)-\left(i^{N}(x,\right. \\
\left.y)+f^{N}(x, y)\right) .
\end{gathered}
$$

Step 11.: Maximum value of $S(x, y)$ gives that $x$ and $y$ are strongest players than the others.

\section{Concluding Remarks}

Graph theory is an enjoyable playground for the research of proof techniques in discrete mathematics. There are many applications of graph theory in different fields. We have introduced the concepts of the bipolar neutrosophic competition graphs. We have described an application of $m$-step bipolar neutrosophic competition graphs in different level of games with the help of an algorithm. We aim to extend our research work to (1) Bipolar fuzzy rough graphs; (2) Bipolar fuzzy rough hypergraphs, (3) Bipolar fuzzy rough neutrosophic graphs, and (4) Decision support systems based on bipolar neutrosophic graphs.

Acknowledgement. The authors are highly thankful to Executive Editor and the referees for their valuable comments and suggestions. 


\section{REFERENCES}

[1] Akram, M., "Bipolar fuzzy graphs", Information Sciences, 181:24(2011), 5548-5564.

[2] Akram, M., "Single-valued neutrosophic planar graphs", International Journal of Algebra and Statistics, 5:2(2016), 157-167.

[3] Akram, M. and Sarwar, M., "Novel multiple criteria decision making methods based on bipolar neutrosophic sets and bipolar neutrosophic graphs", Italian journal of pure and applied mathematics, 38(2017), 1-24.

[4] Akram, M. and Shahzadi, S., "Neutrosophic soft graphs with application", Journal of Intelligent \& Fuzzy Systems, 32(1) (2017), 841-858.

[5] Al-Shehrie, N.O. and Akram, M., "Bipolar fuzzy competition graphs", Ars Combinatoria, 121(2015), 385-402.

[6] Atanassov, K., "Intuitionistic fuzzy sets", Fuzzy Sets and Systems 20:1(1986), 87-96.

[7] Bhattacharya, P., "Some remark on fuzzy graphs", Pattern Recognition Letters, 6(1987), 297-302.

[8] Broumi, S., Talea, M., Bakali, A. and Smarandache, F., "On bipolar single valued neutrosophic graphs", New Trends in Neutrosophic Theory and Applications, (2016), 203-221.

[9] Cho, H.H., Kim, S.-R. and Nam, Y., "The m-step competition graph of a digraph", Discrete Applied Mathematics, 105(2000), 115-127.

[10] Cohen, J.E., "Interval graphs and food webs: a finding and a problems", Document 17696PR, RAND Corporation, Santa Monica, CA, (1968).

[11] Deli, I., Ali, M. and Smarandache, F., "Bipolar neutrosophic sets and their applications based on multi-criteria decision making problems", In Advanced Mechatronic Systems (ICAMechS), International Conference IEEE, (2015), 249-254.

[12] Kauffman, A., Introduction a la theorie des sousemsembles flous, Masson et cie Paris, (1973).

[13] Nasir, M., Siddique, S. and Akram, M., "Novel properties of intuitionistic fuzzy competition graphs", Journal of Uncertain Systems, 2:1(2017), 49-67.

[14] Rosenfeld, A., "Fuzzy graphs", Fuzzy Sets and their Applications (L.A.Zadeh, K.S.Fu, M.Shimura, Eds.), Academic Press, New York, (1975), 77-95.

[15] Samanta, S. and Pal, M., "Fuzzy $k$-competition graphs and $p$-competition fuzzy graphs", Fuzzy Information and Engineering, 5(2013), 191-204.

[16] Samanta, S., Akram, M. and Pal, M., "m-step fuzzy competition graphs", Journal of Applied Mathematics and Computing, 47(2015), 461-472.

[17] Sarwar, M. and Akram, M., "Novel concepts of bipolar fuzzy competition graphs", Journal of Applied Mathematics and Computing, (2016), DOI 10.1007/s12190-016-1021-z.

[18] Smarandache, F., "Neutrosophic set-a generalization of the intuitionistic fuzzy set", Granular Computing, 2006, IEEE International Conference, (2006), 38-42, DOI:10.1109/GRC 1635754.

[19] Smarandache, F., "A geometric interpretation of the neutrosophic set- A generalization of the intuitionistic fuzzy set", Granular Computing, (GrC), 2011 IEEE International Conference, (2011), 602-606, DOI:10.1109/GRC.2011.6122665.

[20] Smarandache, F., "Types of neutrosophic graphs and neutrosophic algebraic structures together with their applications in technology", Seminar, Universitatea Transilvania din Brasov, Facultatea de Design de Produs si Mediu, Brasov, Romania, 06 June (2015).

[21] Smarandache, F., "A unifying field in logics: neutrosophic logic. neutrosophy, meutrosophic set, neutrosphic probability: neutrosophic logic. neutrosophy, neutrosophic set, neutrosophic prbability", Inifinite Study, (2005).

[22] Wang, H., Smarandache, F., Zhang, Y. and Sunderraman, R., "Single valued neutrosophic sets", Multisapace and Multistructure, 4(2010), 410-413.

[23] Wu, S.Y., "The compositions of fuzzy digraphs", Journal of Research in Education Science, 31(1986), 603-628.

[24] Ye, J., "Single-valued neutrosophic minimum spanning tree and its clustering method", Journal of Intelligent Systems, 23:3(2014), 311-324.

[25] Ye, J., "A multicriteria decision-making method using aggregation operators for simplified neutrosophic sets", Journal of Intelligent \& Fuzzy Systems, 26:5(2014), 2459-2466. 
[26] Zadeh, L.A., "Fuzzy sets", Information and Control, 08:3(1965), 338-353.

[27] Zadeh, L.A., "Similarity relations and fuzzy orderings", Information Science, 3:2(1971), $177-200$.

[28] Zhang, W.-R., "Bipolar fuzzy sets and relations: a computational framework for cognitive modeling and multiagent decision analysis", In: Proceedings of IEEE Conference Fuzzy Information Processing Society Biannual Conference, (1994), 305-309. 